\title{
A Slow Short-Term Depression at Purkinje to Deep Cerebellar Nuclear Neuron Synapses Supports Gain-Control and Linear Encoding over Second-Long Time Windows
}

\author{
Christine M. Pedroarena ${ }^{1,2}$ \\ ${ }^{1}$ Department for Cognitive Neurology, Hertie-Institute for Clinical Brain Research, 72076 Tübingen, Germany, and ${ }^{2}$ Systems Neurophysiology, \\ Werner Reichardt Center for Integrative Neuroscience, University Tübingen, 72076 Tübingen, Germany
}

Modifications in the sensitivity of neural elements allow the brain to adapt its functions to varying demands. Frequency-dependent short-term synaptic depression (STD) provides a dynamic gain-control mechanism enabling adaptation to different background conditions alongside enhanced sensitivity to input-driven changes in activity. In contrast, synapses displaying frequency-invariant transmission can faithfully transfer ongoing presynaptic rates enabling linear processing, deemed critical for many functions. However, rigid frequency-invariant transmission may lead to runaway dynamics and low sensitivity to changes in rate. Here, I investigated the Purkinje cell to deep cerebellar nuclei neuron synapses (PC_DCNs), which display frequency invariance, and yet, PCs maintain background activity at disparate rates, even at rest. Using protracted PC_DCN activation (120 s) to mimic background activity in cerebellar slices from mature mice of both sexes, I identified a previously unrecognized, frequencydependent, slow STD (S-STD), adapting IPSC amplitudes in tens of seconds to minutes. However, after changes in activation rates, over a behavior-relevant second-long time window, S-STD enabled scaled linear encoding of PC rates in synaptic charge transfer and DCN spiking activity. Combined electrophysiology, optogenetics, and statistical analysis suggested that S-STD mechanism is input-specific, involving decreased ready-to-release quanta, and distinct from faster short-term plasticity (f-STP). Accordingly, an S-STD component with a scaling effect (i.e., activity-dependent release sites inactivation), extending a model explaining PC_DCN release on shorter timescales using balanced f-STP, reproduced the experimental results. Thus, these results elucidates a novel slow gain-control mechanism able to support linear transfer of behavior-driven/learned PC rates concurrently with background activity adaptation, and furthermore, provides an alternative pathway to refine PC output.

Key words: background activity; gain modulation; short-term memory; short-term plasticity; sustained activity; synaptic transmission

\section{Significance Statement}

The brain can adapt to varying demands by dynamically changing the gain of its synapses; however, some tasks require ongoing linear transfer of presynaptic rates, seemingly incompatible with nonlinear gain adaptation. Here, I report a novel slow gain-control mechanism enabling scaled linear encoding of presynaptic rates over behavior-relevant time windows, and adaptation to background activity at the Purkinje to deep cerebellar nuclear neurons synapses (PC_DCNs). A previously unrecognized PC_DCNs slow and frequency-dependent short-term synaptic depression (S-STD) mediates this process. Experimental evidence and simulations suggested that scaled linear encoding emerges from the combination of S-STD slow dynamics and frequency-invariant transmission at faster timescales. These results demonstrate a mechanism reconciling rate code with background activity adaptation and suitable for flexibly tuning PCs output via background activity modulation.

\section{Introduction}

Changes in the sensitivity or gain of neural structures is a general principle that allows the brain to adapt to varying sensory, motor, emotional, or cognitive demands (Silver, 2010; Whitmire and Stanley, 2016). One mechanism supporting gain adaptation

\footnotetext{
Received Aug. 27, 2019; revised Apr. 21, 2020; accepted May 23, 2020.

Author contributions: C.M.P. designed research; C.M.P. performed research; C.M.P. analyzed data; C.M.P.

wrote the first draft of the paper; C.M.P. edited the paper; C.M.P. wrote the paper.

The author declares no competing financial interests.
}

is use-dependent short-term synaptic depression (STD), where high levels of activity lead to lower synaptic strength, thereby

I thank Cornelius Schwarz for comments on a version of this manuscript and support for this research, alongside that from the Cognitive Neurology Department; Ute Grosshennig and Ursula Pascht for technical assistance; all members of the Cognitive Neurology Department for comments and helpful discussions; as well as the editors and the anonymous reviewers for helpful comments and suggestions.

Correspondence should be addressed to Christine M. Pedroarena at christine.pedroarena@uni-tuebingen.de. https://doi.org/10.1523/JNEUROSCI.2078-19.2020

Copyright $\odot 2020$ the authors 
dynamically adapting the output while enhancing the sensitivity to changes in presynaptic activity (Abbott et al., 1997; Tsodyks and Markram, 1997). The latter is because changes in synaptic strength lag behind the change in presynaptic activity, such that for short time windows, in the millisecond range for fast-STDs (Zucker and Regehr, 2002), the output becomes proportional to the new rate, before readapting. This process produces brief transient signals informative about the timing and proportion of the initial change in rate (Abbott et al., 1997). However, beyond this brief window, STD filters the synaptic output preventing faithful transfer of complete presynaptic rate profiles, which may hold important information.

Such is the case of the cerebellar cortex principal neurons, the GABAergic Purkinje cells (PCs), which display changes in rate associated to cerebellar-controlled behaviors (e.g., eye-blinks, saccades, and whisks) extending beyond the referred STD time window, considered critical for representing and controlling specific behavioral parameters (e.g., Catz et al., 2008; Herzfeld et al., 2015; Ten Brinke et al., 2017; Romano et al., 2018). Suitably, the synapses of the PCs to their main target, the deep cerebellar nuclei neurons, the cerebellar output stage (PC_DCNs), exhibit frequency-invariant transmission supporting linear encoding of PC rates (Turecek et al., 2016). Mechanistically, PC_DCN frequency invariance is mediated by a developmentally regulated synaptotagmin-7-dependent process, possibly synaptic facilitation (Turecek et al., 2017) counteracting frequency-dependent fast-STD (Pedroarena and Schwarz, 2003). Functionally, linear encoding has been widely reported in the cerebellum, including the cerebellar nuclei (Arenz et al., 2008; Herzfeld et al., 2015; S. Chen et al., 2016; Hong et al., 2016; Jelitai et al., 2016; Abbasi et al., 2017; Dugué et al., 2017; Ten Brinke et al., 2017; Payne et al., 2019; but see Halverson et al., 2015), although alternative coding strategies could (co)exist (De Zeeuw et al., 2011). Reasonably, the latter studies focused on time windows matching usual behaviors (i.e., ranging from subsecond to few seconds).

Yet, PCs discharge simple spikes almost continuously at disparate rates diverging by as much as $100 \mathrm{~Hz}$, even in awake animals at rest and within the same region (Thach, 1968; De Zeeuw et al., 1995; Bryant et al., 2009; Xiao et al., 2014; Zhou et al., 2014; Laurens and Angelaki, 2017). Therefore, PC signals associated with particular behaviors consist of transitory changes in rate embedded in the background activity. PC background activity has received much less attention than the behavior-driven signals, although it is altered in some cerebellar-associated diseases (Rinaldo and Hansel, 2010; Tsai et al., 2012). Differences in intrinsic properties and ongoing PC inputs explain PC background rates diversity and variability (Ozden et al., 2012; Zhou et al., 2014; Jelitai et al., 2016). This suggests that PC background activity might be a controlled variable, potentially moldable via PC intrinsic plasticity (Belmeguenai et al., 2010; Shim et al., 2017; Grasselli et al., 2020), plastic changes of the upstream cerebellar circuits (Gao et al., 2012), and/or context-dependent changes in the ongoing activity of PCs numerous inputs. However, PC background activity represents a challenge for frequency-invariant synapses because the postsynaptic effect of behavior-driven/learned changes in PC rate could be obscured by the wide span of background rates from converging PCs (Abbott et al., 1997), as for keeping DCNs within their workingfiring range.

Expanding the computational power of PC_DCNs by coexpression of different forms of short-term plasticity (STP) (Magleby, 1973; MacLeod et al., 2007; Turecek et al., 2016; Doussau et al., 2017) could solve this conundrum; however,
PC_DCN-STP has not been previously explored at timescales matching background activity. This study, by closing this gap, identified a novel mechanism supporting concurrent rate code and background activity adaptation and potentially useful for tuning PC output.

\section{Materials and Methods}

The animal protocol was reviewed and approved by an independent local committee and the Regional Council of Tübingen and conducted according to the standards of German law and the Society for Neurosciences.

Cerebellar slices preparation. Cerebellar slices from C57BL/6 or L7ChR2-eYFP (Chaumont et al., 2013) mice (either sex, P21-P32), deeply anesthetized with ketamine $(150 \mathrm{mg} / \mathrm{kg})$, were prepared using a vibratome (Leica Microsystems) and ACSF (containing the following, in $\mathrm{mM}$ : 125.5 NaCl, $2.5 \mathrm{KCl}, 1.3 \mathrm{NaH}_{2} \mathrm{PO}_{4}, 1 \mathrm{MgCl}_{2}, 26 \mathrm{NaHCO}_{3}, 20$ glucose, $1.5 \mathrm{CaCl}_{2}$ ), bubbled with $95 \% \mathrm{O}_{2} / 5 \% \mathrm{CO}_{2}$ and, warmed at $26^{\circ} \mathrm{C}$. Slices were first stored for $30 \mathrm{~min}$ at $36^{\circ} \mathrm{C}$ and afterward at room temperature in the same solution. The same solution was used for recordings. A set of experiments using male and female C57 BL/6 adult mice (P55 to P72) was conducted using similar procedures.

Patch-clamp recordings and extracellular stimulation. Whole-cell voltage-clamp, or cell-attached recordings were made from large $(>20$ $\mu \mathrm{m})$ DCNs in the lateral or interpositus nuclei of cerebellar slices maintained at $36 \pm 0.5^{\circ} \mathrm{C}$ using an Axoclamp1D-amplifier (Molecular Devices). For voltage-clamp recordings, the electrode solution contained the following (in mM): $145 \mathrm{CsCl}, 10 \mathrm{~K}-\mathrm{HEPES}, 0.5 \mathrm{EGTA}, 0.4 \mathrm{NaGTP}$, $4.8 \mathrm{~K}-\mathrm{ATP}, 2.5 \mathrm{MgCl}_{2}, 0.1 \mathrm{CaCl}_{2}, 5 \mathrm{QX} 314$. The $\mathrm{pH}$ was adjusted to 7.3 with $\mathrm{CsOH}$. In a small set of experiments $(n=5)$, electrodes were filled with a solution containing the following (in $\mathrm{mM}$ ): $134 \mathrm{~K}$-gluconate, 6 $\mathrm{KCl}, 10 \mathrm{~K}-\mathrm{HEPES}, 0.1$ EGTA, $0.3 \mathrm{NaGTP}, 2 \mathrm{~K}-\mathrm{ATP}, 10$ phosphocreatine, $2 \mathrm{MgCl}_{2}$, $5 \mathrm{QX}-314$, and cells were clamped at $0 \mathrm{mV}$. The results were similar to the ones obtained with the CsCl-based solution, and thus later pooled together for analysis. Series resistance was adjusted up to $75 \%$, and recordings were discarded when series resistance (usually controlled before and after trials) changed $>20 \%$. For cell-attached recordings, the pipettes were filled with ACSF. Recordings were digitized $(12.5 \mathrm{kHz})$, and stored using programmable software (Spike 2, CED).

To stimulate PC axons, a pair of tungsten microelectrodes (Frederick Haer) was located in the white matter surrounding the cerebellar nuclei. Biphasic current pulses (100 $\mu$ s each phase or $100 / 50 \mu \mathrm{s}, 2-100 \mu \mathrm{A}$, usually $<25 \mu \mathrm{A}$ ) were delivered using a constant current unit (stimulus isolator, WPI) triggered by the Spike 2 software. The electrodes were oriented to minimize the stimulation current necessary to activate axons in the white matter and to reduce the possibility of intranuclear stimulation. Single stimuli for evaluating IPSCs under control conditions were delivered at 0.1 or $0.05 \mathrm{~Hz}$. To simulate PC tonic background firing, PC axons were repetitively stimulated at $10,29.5$, or $67 \mathrm{~Hz}$ for at least $2 \mathrm{~min}$, interleaving a pause of at least $10 \mathrm{~min}$ between trials and in variable order. To investigate the effect of irregular PC activation, Poisson-like activation patterns with mean frequencies 10,30 , or $70 \mathrm{~Hz}$ were delivered using Spike 2 software.

For opto-genetic (OG) stimulation of PC axons and terminals light pulses $(0.5-2 \mathrm{~ms}, 470 \mathrm{~nm})$ from a LED (CoolLed), triggered by the Spike 2 software were delivered through the microscope objective to cerebellar slices from L7-ChR2-eYFP mice (Chaumont et al., 2013). The location of the spot of light was adjusted before recording using the microscope diaphragm in the light path. The time course of OG and combined electrical stimulation (ES) IPSCs could be very similar, suggesting similar mechanism of neurotransmitter release. However, in some experiments (even when the spot of light was centered in the white matter), the OG IPSCs presented steps in the rising and decaying phase and were on average slower. This observation may reflect desynchronized activation due to variable latency for triggering spikes in different axons or terminals.

Drugs. Kynurenate (3-5 mm, Tocris Bioscience), a broad-spectrum antagonist of ionotropic glutamate receptors, was systematically applied to the ACSF, to isolate pharmacologically the response of PC synapses 
from the potential glutamatergic responses evoked by activation of mossy and climbing fiber collaterals in the white matter. In addition, in specific experiments exploring DCN responses, strychnine ( $1 \mu \mathrm{M}$, Sigma Millipore), a glycine receptor antagonist, and CGP 55845 (3 $\mu \mathrm{M}$, Tocris Bioscience), a $\mathrm{GABA}_{\mathrm{B}}$ receptor antagonist, were applied to the bath. These results were similar to those without these two blockers, and thus later pooled together for analysis.

Experimental design and statistical analysis. Programmable software (Spike 2, CED) was used to automatically calculate IPSC peak amplitude as the difference between the average baseline (measured for $300 \mu \mathrm{s}$ before each stimulus) and the IPSC peak. For trains with interpulse intervals $\leq 10 \mathrm{~ms}$, the baseline was calculated by fitting an exponential decay to the preceding IPSC. For analysis of repetitive IPSCs, the IPSC peak amplitude was normalized to the amplitude of corresponding control IPSCs evoked at low frequency immediately before each train of stimuli. For experiments using protracted stimulation, only data from experiments with at least two different stimulating frequencies of either regular or Poisson-like patterns were analyzed. In some cases, and apparently erratically, responses to trains of stimuli displayed after a phase of depression a transient rebound in amplitude and later a depression as usual. As it is not clear whether this is a true synaptic phenomenon, these traces were not included for the analysis of changes in IPSC amplitudes along the train.

For calculating the charge transferred (Q) during trains of IPSCs, the stimulus artifacts (which terminated before the foot of the IPSCs) were digitally removed and the difference in area between an ROI of the trace (e.g., high frequency stimulation [HFS]) and control (no stimulation) was calculated using Spike 2 software (CED). The values were normalized to the charge transferred by the corresponding control IPSC and expressed per unit of time. Because the level of depression varied in different neurons, for normalization to the steady-state values of IPSCs, I used the mean charge transferred by the corresponding IPSCs at steady state.

To investigate slow and frequency-dependent short-term synaptic depression (S-STD) input specificity, I combined ES and OG stimulation of PC axons. Using optical stimulation alone was not practical. First, contrary to the reliable activation of PC somas recorded from the same slices, evoking PC_DCN IPSCs by directing the spot of light toward terminals and axons over the recorded DCN soma or the neighboring white matter required higher intensity than for activating PC somas, but using too high light intensity could lead to transmission block. For this reason, the light intensity was kept submaximal throughout the OG experiments. Second, OG stimulation using repetitive stimuli at high frequencies resulted in stronger depression of PC_DCN IPSCs than using ES, as observed earlier, presumably due to channelrhodopsin inactivation (Najac and Raman, 2015). Third, block of transmission occurred sometimes after repeated OG activation at high or low frequency without evident alteration of the transmission evoked by electrical activation of PC axons, suggesting long-lasting channelrhodopsin inactivation. Therefore, to test input specificity, I assessed the effect of $120 \mathrm{~s}$ of repetitive ES on OG IPSCs and ES IPSCs, with intensities adjusted to evoke larger OG IPSCs. Assuming larger OG IPSCs resulted from a larger number of OG than ES activated synapses, a subgroup of synapses was activated exclusively (or almost exclusively) by the light pulses and, thus, spared during the $120 \mathrm{~s}$ of ES.

Analysis of changes in the inverse squared coefficient of variation $\left(\mathrm{CV}^{-2}\right)$ of IPSCs was used as described previously (Pedroarena and Schwarz, 2003) to assess the presynaptic or postsynaptic locus of the STP and possible mechanisms. Briefly, assuming the mean amplitude of the IPSCs $(M)$ can be explained by the product of the number of releasable quanta $(N)$, the probability of each quanta to be released $(p)$, and the mean postsynaptic response to one quantum $(q)$, the $\mathrm{CV}^{-2}\left(=\frac{M^{2}}{\sigma^{2}}=\frac{N p}{1-p}\right)$, where $\sigma^{2}$ is the variance around $M$, estimates the variability of synaptic events (Bekkers and Stevens, 1990; Malinow and Tsien, 1990). Plots of $\mathrm{CV}^{-2}$ versus the corresponding $M$ values (as ratio of controls) are useful to differentiate presynaptic from postsynaptic mechanisms of synaptic plasticity because the $\mathrm{CV}^{-2}$ is mostly insensitive to $q$. Furthermore, as $\mathrm{CV}^{-2}$, in contrast to $M$, is differentially affected by changes in $N$ or $p$, this method provides an indication of possible presynaptic basis of the changes in $M$, as confirmed at PC_DCNs previously (Pedroarena and Schwarz, 2003). For illustration, Figure $5 C$ (inset) shows the expected $\mathrm{CV}^{-2}$ versus $\mathrm{M}$ values as calculated from changes in $N$, $p$, or $q$. At least 100 IPSCs obtained under steady-state conditions (Control, 0.1 or $0.05 \mathrm{~Hz}$, or after IPSC depression stabilized using 10,29.5, or $67 \mathrm{~Hz}$ of background frequency $[\mathrm{BF}]$ ) were used for this analysis.

Programmable software (Spike 2, CED; Igor, Wavemetrics; and Sigma Plot SPSS, IBM) was used for data processing and to carry statistical analysis. Data were tested for normality (using the Shapiro-Wilcoxon test) and equal variance. For populations that passed or failed the normality and equal variance tests, parametric (Student two tailed $t$ tests, unpaired or paired according to the conditions) or nonparametric tests (Mann-Whitney rank sum test or Wilcoxon signed rank sum tests) were used, respectively. Data are presented as mean \pm SEM, unless otherwise noted. Sample sizes were not predetermined but are reported together with the type of test used and the corresponding $p$ values.

Simulations. To simulate synaptic transmission and STP at PC_DCN synapses as a starting point, I used a version of a previously published model, which has been shown to correctly predict the synaptic output to repetitive activation of PC_DCN synapses with second long trains (Turecek et al., 2016, 2017). Specifically, two pools of ready to be released vesicles (RRPs), differing in release probability, recovery rate, and presence or not of facilitation of release, were required by this model to explain the IPSCs initial fast depressing phase, the sustained release during hundreds of events, and the frequency invariance observed experimentally. As noted previously, the two different pools could correspond to two different states of a single pool (Neher and Sakaba, 2008); and in this scenario, facilitation could, at least in part, result from expedited transition to the mature state (Pan and Zucker, 2009; Miki et al., 2016). However, here for simplicity and comparison with previous results at this synapse, I adopted the parallel model. The present version is referred here as the D + F model. Briefly, the initial conditions are determined by the number of ready to be released vesicles in each pool (RRPA and RRPB), and the intrinsic probability of each vesicle class to be released ( $\operatorname{Pr} A, \operatorname{PrB})$, plus the level of total facilitation for the Pool B (F1 and F2). The total number of vesicles released $(\mathrm{P})$ in response to a stimulus $\left(\mathrm{S}_{\mathrm{p}}\right)$ is the linear sum of Pools $\mathrm{A}$ and $\mathrm{B}$ released vesicles (PA and $\mathrm{PB}$, respectively), and it is assumed that the postsynaptic response to neurotransmitter is identical for both pools. The total number of vesicles released from each pool with each stimulus is given by the product of the corresponding RRP and $\mathrm{Pr}$ or Pr plus the corresponding level of facilitation (F). Therefore,

$$
P_{p}=R R P A_{p} \times P r A+R R P B_{p} \times(P r B+F 1+F 2)
$$

Although the precise mechanism(s) underlying synaptic facilitation (and probability of release) is not yet completely understood (Valera et al., 2012; Turecek et al., 2017; Chang et al., 2018), here following others, facilitation of release was simulated by an increase in the release probability, in particular, an increase in PrB. The total amount of facilitation after each stimulus is given by the addition of a constant amount $(f)$ added right after each stimulus plus the remaining facilitation, which decays to the initial condition with time constant Tauf during the interval between stimuli. $F_{p+}$ is as follows:

$$
\left.F_{p+}=f+F_{p-1} \times e^{\left(-{ }^{-I n t} /\right. \text { Tauf }}\right)
$$

where Int is the time interval between the present stimulus and the preceding one. $f$ is added right after each stimulus; thus, the amount of facilitation added to $\operatorname{PrB}$ at the moment of the stimulus $p$ is $=\left(F_{p+}-f\right)$.

Evidence from previous studies using mice younger than P21 suggested that a different rapid and short-lasting facilitation could occur at these synapses (Pedroarena and Schwarz, 2003). Thus, two facilitation factors (F1 and F2) were included following the same scheme, but one of them was constrained to have Tauf $<10-15 \mathrm{~ms}$. The total facilitation added to PrB is the sum of these two factors. However, fits to the model 
did not show an important contribution of the short-lasting facilitation component under the conditions here explored.

During the interval between stimuli, the RRP of each vesicle pool recovers to the initial condition following a time constant $\left(\mathrm{Tau}_{\mathrm{RA}}\right.$ or $\mathrm{Tau}_{\mathrm{RB}}$ ). Therefore, the number of available vesicles at Stimulus ${ }_{\mathrm{p}}$ for each pool is given by the following:

$$
\begin{aligned}
R R P_{p=} & R R P_{p-1} \times(1-P r)+\left(R R P_{0}-\left(R R P_{p-1}\right.\right. \\
& \times(1-(P r+F)))) \times\left(1-e^{- \text {Int }} / \text { TauR }\right)
\end{aligned}
$$

Accordingly, depression in this model results from depletion of RRPs, when release exceeds the number of vesicles recovered, and therefore is larger, the shorter the interval between stimuli. The simulations and fits were conducted using IgorPro (Wavemetrics). The parameters were repeatedly adjusted by fitting the model to the normalized peak amplitude of averaged PC_DCN IPSCs evoked by stimulation trains of 100 events delivered at different frequencies $(10-200 \mathrm{~Hz})$. The set of parameters that best fit all conditions was used to further simulate the responses to protracted stimulation $\left(\mathrm{RRPA}_{0}=7, \mathrm{RRPB}_{0}=25, \operatorname{PrA}=\right.$ $0.098, \operatorname{PrB}=0.017$, Taur $\mathrm{A}=12$, TaurB $=0.5, \mathrm{f} 1=0.0005$, TauF1 $=0.007$, $\mathrm{f} 2=0.001$, Tauf $2=0.1$ ).

Because the D $+\mathrm{F}$ model, with or without facilitation, did not explain the slow depression observed with protracted stimulation, it was extended to include a slow-depression component. To this end, I simulated an activity-dependent decrease in the number of "active release sites" according to findings of this study and previous postulates (see Discussion) (Stevens and Wesseling, 1999; Neher, 2010). Because the pool of vesicles A is largely depleted after 100 events, I simulated an activity-dependent decrease in release sites of the Pool B alone. For this purpose, the new model (referred as the SD_RS model) features a number of release sites B (R_SitesB) that initially is identical to RRPB with all release sites occupied by vesicles. After use, some of these sites become unavailable to be refilled, and availability is restored with a time constant TauRSr. The remaining release sites can be either empty or filled, according to the rate of depletion of Pool B. Importantly, the processes affecting remaining release sites are not affected using this implementation. Therefore, although the mechanism of synaptotagmin-7-dependent PC_DCN frequency-invariant transmission may not be completely elucidated (C. Chen et al., 2017), the use of balanced depression and facilitation to simulate frequency invariance is justified. A previous study based on the presence of a PC_DCN tonic current associated to frequencyinvariant depression evoked by repetitive PC_DCN stimulation in recordings from 10 to $20 \mu \mathrm{m}$ DCNs carried at $31^{\circ} \mathrm{C}$ and obtained in slices from young mice postulated pooling and saturation of postsynaptic receptors as a possible mechanisms for frequency independence (Telgkamp et al., 2004). This idea seems unlikely at PC synapses of large DCNs from mature animals at close to physiological temperatures, as used in the present study, because application of TPMPA, a low-affinity competitive GABA-A antagonist (which relieves postsynaptic receptor saturation and pooling) does not prevent the developmentally dependent frequency invariance of PC_DCN IPSCs (Turecek et al., 2016). Moreover, under the here used conditions and type of synapses, I (and others) did not find significant tonic currents (Najac and Raman, 2015; Turecek et al., 2016).

To avoid making assumption about the molecular nature of the activity-dependent mechanism that leads to decrease in "active release sites," and following empirical calculations, the amount of release sites inactivated per time unit (NdecRS) was calculated as follows:

$$
N d e c R S=A R S B \times\left(1-e^{-F r e q} / F_{-} R S\right.
$$

where $A R S B$ is the maximal number of release sites that can become unavailable, $F \_R S$ is a parameter determining the frequency dependence of this process. The inverse of the preceding interval was used here to calculate Freq; thus, we assume that the local level of activity determines this parameter. Alternatively, the averaged stimulating frequency over $\times$ time preceding each stimulus could be used to simulate a process depending on global level of activity; but under the conditions here tested, good fits were found using instantaneous frequency. The number of release sites inactivated per stimulus is the value of NdecRS calculated at each stimulus time point, scaled by the preceding time interval.

The unavailable release sites recover following:

$$
R S r_{p}=\left(R \_ \text {Sites } B_{0}-R \_ \text {Sites } B_{p-1}\right) \times\left(1-e^{-I n t} / \text { TauRst }\right),
$$

Where $R S r_{p}$ represents the number of "active release sites" of Pool B recovered during the preceding interval, $R_{-} S i t e s B_{0}$ is the initial number of active release sites, $R \_$Sites $B_{p-1}$ is the number of active release sites available at the precedent event, and TauRSr is the time constant of release sites recovery.

Therefore, the number of "active release sites" available at the time of each stimulus is as follows:

$$
R_{-} \text {Sites } B_{p}=R \_ \text {Sites } B_{p-1}+R S r_{p}-\left(N d e c R S_{p} \times \text { Int }\right)
$$

In the SD_RS model, $R \_$Sites $B_{p}$ substitutes for $R R P_{0}$ in Equation 3 for Pool B.

To estimate the new parameters corresponding to the SD_RS model, the normalized peak amplitude of averaged PC_DCN IPSCs evoked by stimulation trains of $120 \mathrm{~s}$ delivered at different frequencies was used to fit the model in Igor. The set of parameters that best fit all conditions was used to evaluate the response of the model to transient changes in stimulating frequency from steady state $\left(A R S B=0.47, F \_R S=29\right.$; $T a u R S r=30$ ). Additionally, I tested the ability of the model to explain the responses from different sets of experiments using a different stimulation paradigm (i.e., a sustained change in stimulating frequency in the same file) (Figs. 7 and 8).

\section{Results}

\section{S-STD of PC_DCN IPSCs}

To explore the response of PC_DCN synapses to sustained activity, I used cerebellar slices from juvenile (P21-P32) or adult (P55-P72) mice maintained under conditions like those in situ (i.e., temperature: $36.5 \pm 0.5^{\circ} \mathrm{C}$ and extracellular $\mathrm{CaCl}_{2}$ concentration: $1.5 \mathrm{~mm}$ ), as variations in these parameters and the developmental stage affect synaptic transmission and STP (Borst, 2010). Whole-cell patch-clamp or cell-attached recordings were obtained from large DCNs $(>20 \mu \mathrm{M})$ in the lateral or the interpositus nuclei because this group of DCNs can be unambiguously identified as projecting, putative glutamatergic DCNs, while smaller DCNs may be part of different subgroups, potentially displaying different synaptic properties (Uusisaari et al., 2007; Uusisaari and Knopfel, 2008; Najac and Raman, 2015). To mimic the persistent discharge of PCs, I used protracted ES of $\mathrm{PC}$ axons in the surrounding white matter $(\geq 2 \mathrm{~min})$ at three different stimulating background frequencies (BF: 10, 29.5, and $67 \mathrm{~Hz}$ ), which are within the PCs firing rate range in awake animals at rest (Thach, 1968; Zhou et al., 2014).

The responses to 2-min-long trains of stimuli confirmed an initial phase of rapid decrease in phasic IPSC peak amplitude with successive events, which slowed down and leveled after tens of events (Telgkamp and Raman, 2002; Pedroarena and Schwarz, 2003; Turecek et al., 2016) (representative traces from one neuron in Fig. $1 A$, and mean normalized peak amplitude as a function of time across neurons in Fig. $1 B$ ). However, the use of protracted stimulation unveiled a different slow phase of decay in IPSC peak amplitude ensuing the fast one, with time constants ranging from 18 to $47 \mathrm{~s}$, most evident by analyzing the results in a compressed timescale (Fig. $1 B$, insets, fits using double exponential decay functions). Furthermore, the average IPSC amplitude at the end of the 2 min stimulation periods decreased with 

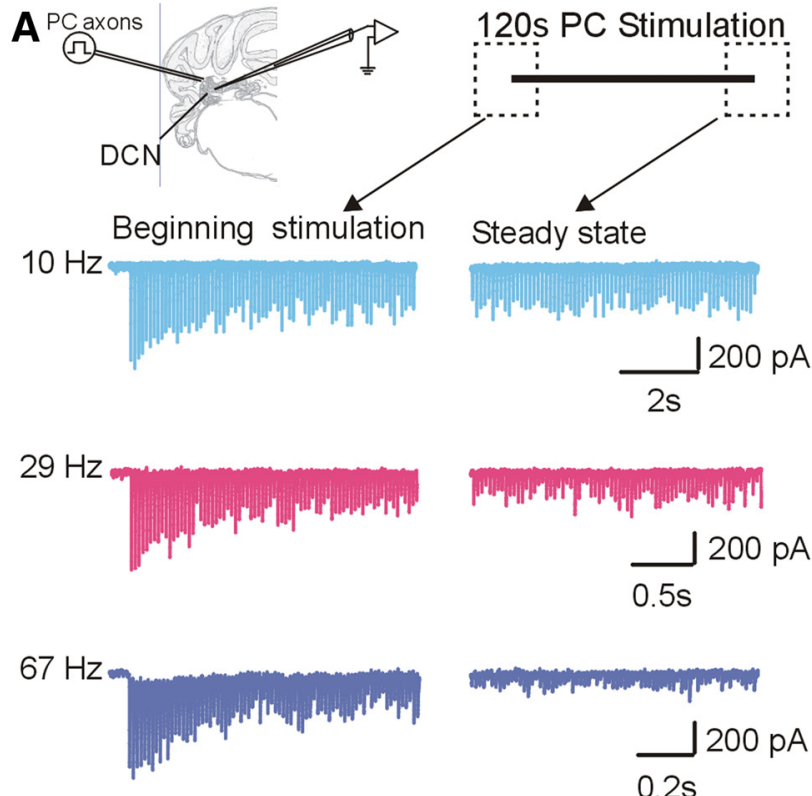

E

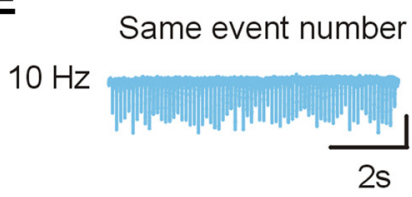

$29 \mathrm{~Hz}$

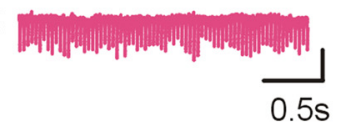

$67 \mathrm{~Hz}$

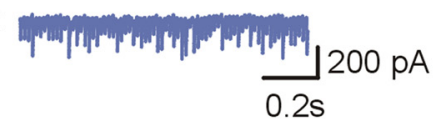

F IPSC peak amplitude

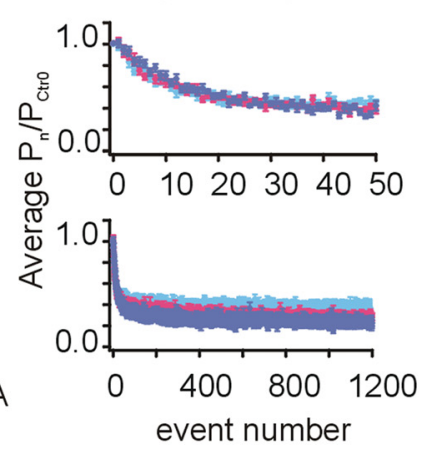

B IPSC peak amplitude
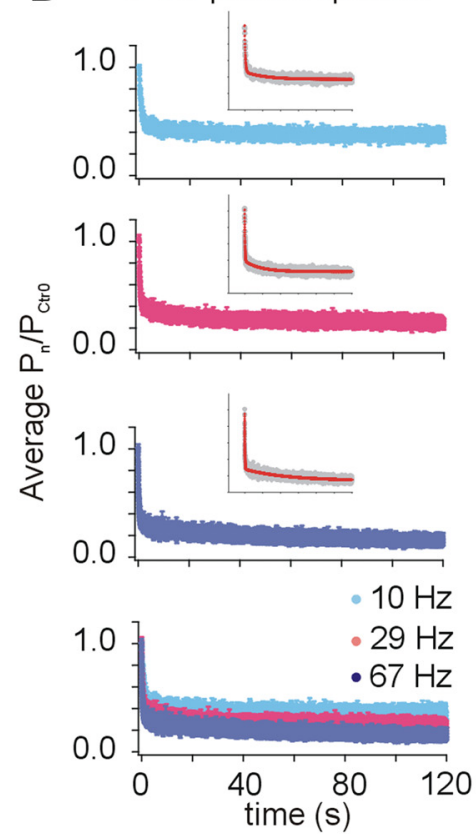

G

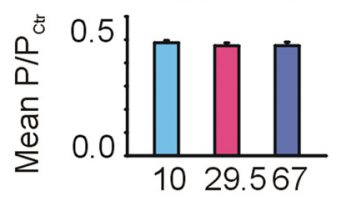

Events 1000-1100

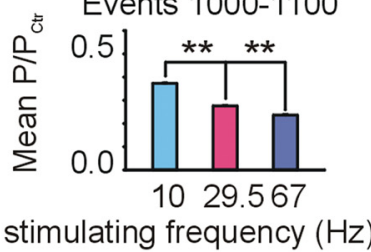

C IPSC peak amplitude

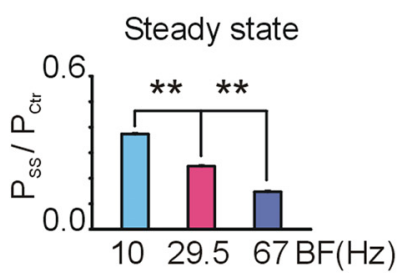

D

Ind. Means SS
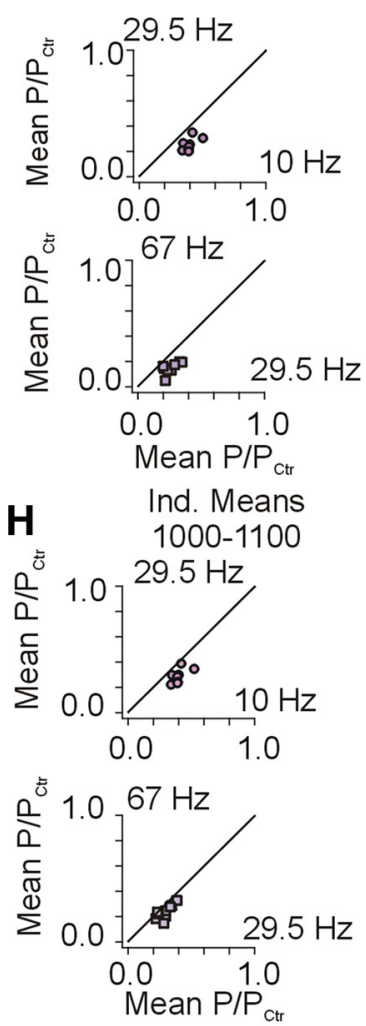

Figure 1. Protracted PC stimulation unveils an S-STD. A, Top, Sketch of experimental configuration and stimulation paradigm. Bottom, Typical examples of IPSCs from the same neuron evoked by 2 min trains of stimuli applied to $P C$ axons at the frequencies (BF) indicated on the left, at the beginning (left), and at the end of stimulation (right). Note the different time scales. $\boldsymbol{B}$, Top to bottom, Mean normalized IPSC peak amplitude as a function of time for BFs $10 \mathrm{~Hz}$ (light blue, $n=11$ ), $29.5 \mathrm{~Hz}$ (red, $n=9$ ), and $67 \mathrm{~Hz}$ (dark blue, $n=9$ ), and all three plots overlaid (symbols represent mean \pm SEM) illustrate two phases of decay. Color code applies to all figures, unless otherwise stated. The double exponential decay fits in the insets: fast tau: $0.81 \pm 0.04,0.41 \pm 0.012$, and $0.33 \pm 0.007 \mathrm{~s}$ and slow tau: $23 \pm 1.9,18 \pm 0.5$, and $47 \pm 1.2 \mathrm{~s}$ for 10, 29.5, and $67 \mathrm{~Hz}$ BFs, respectively. C, Average normalized steady-state phasic IPSCs amplitude (IPSC $C_{S S}$ ) versus BF (averages calculated over the last 100 events of the data plotted in $B ; 0.37 \pm 0.002,0.25 \pm 0.002$, and $0.15 \pm 0.002$ for $10,29.5$, and $67 \mathrm{~Hz}$ BFs, respectively: 10 versus $29.5 \mathrm{~Hz}$ [Mann-Whitney rank sum test] and $29.5 \mathrm{vs} 67 \mathrm{~Hz}$ [t test], $p<0.001$ for both comparisons). D, Results from individual neurons illustrate decreasing mean IPSC $C_{S S}$ with increasing BF (averages over 100 events). Top, IPSC amplitude for 29.5 versus $10 \mathrm{~Hz}$. Bottom, IPSC amplitude for 67 versus $29.5 \mathrm{~Hz}$. All points fall below the unity line. $\boldsymbol{E}$, Typical examples from the same neuron in $\boldsymbol{A}$ of IPSCS evoked by the same ordinal stimuli but at different BFs (indicated on the left). The $10 \mathrm{~Hz}$ trace is the same shown in $\boldsymbol{A}$ for comparison. $\boldsymbol{F}$, Summary of IPSC amplitude as in $\boldsymbol{B}$, but as a function of the event number (color labeled as $\boldsymbol{B}$ ). Top, Events 1-50. Bottom, Events 1-1200. $\boldsymbol{G}$, Averages as in $\boldsymbol{C}$, calculated over the events indicated on top of each bar plot. Top, Events 10-50: $0.49 \pm 0.01,0.47 \pm 0.02$, and $0.47 \pm 0.03$, for $10,29.5$, and $67 \mathrm{~Hz}$, respectively; Wilcoxon signed rank sum test: 10 versus $29.5 \mathrm{~Hz}, p=0.115 ; 29.5 \mathrm{versus} 67 \mathrm{~Hz}, p=0.7$. Bottom, Events 1000-1100: $0.37 \pm 0.002,0.276 \pm 0.002$, and $0.236 \pm 0.002$, for $10,29.5$, and $67 \mathrm{~Hz}$, respectively, $t$ test, 10 versus 29.5 and 29.5 versus $67 \mathrm{~Hz}, p<0.001$ for both. $\boldsymbol{H}$, Similar as in $\boldsymbol{D}$, but for mean IPSCs amplitudes calculated over stimuli 1000-1100th for each neuron. Top, $29.5 \mathrm{~Hz}$ versus $10 \mathrm{~Hz}$. Bottom, 67 versus $29.5 \mathrm{~Hz}$. Data are mean \pm SEM. ${ }^{* *} p<0.001$.

increasing stimulating BFs, suggesting divergence from the frequency-invariant phase described before at earlier stages of PC activation trains (Turecek et al., 2016) (Fig. 1B,C). Importantly, the population results reflected the results from single experiments, depicted individually in Figure $1 D$. Although the magnitude of depression varied among neurons, for each case, the normalized steady-state synaptic strength decreased with increasing BFs (mean values: $0.4 \pm 0.02[10 \mathrm{~Hz}]$, vs $0.26 \pm 0.02$ [29.5 Hz], $n=8$, paired $t$ test, $p<0.001 ; 0.26 \pm 0.02[29.5 \mathrm{~Hz}]$ vs $0.15 \pm 0.015[67 \mathrm{~Hz}], n=9$, paired $t$ test, $p<0.001)$. Therefore, protracted activation of PC_DCNs revealed an S-STD, which at steady state appears as frequency-dependent. Because PCs are continuously active, the differences in mean IPSC amplitudes for different BFs (more than double comparing $10 \mathrm{vs} 67 \mathrm{~Hz}$ ) are likely functionally relevant.

To confirm S-STD frequency dependence and to explore its buildup along the stimulating trains, I analyzed the changes in synaptic strength as a function of the event number (Fig. $1 E-H$ ). In agreement with previous studies using similar conditions as here, the IPSC amplitudes initially, over the first 10 events, depressed significantly but on average with slightly lower rates with increasing BFs (Fig. $1 F$, top), while later, after tens of events, the IPSCs evoked with different BFs displayed similar amplitudes (frequency-invariant phase; Fig. $1 F, G$, top plots) (Turecek et al., 
2016). However, after hundreds of events, the IPSC amplitudes started to diverge, indicating dependence to the time interval between stimuli (Fig. $1 F$, bottom). The IPSCs further decayed, but at different rates for different BFs. The time constants of decay calculated after the first 100 events using single exponential fits were $216 \pm 24,612 \pm 21$, and $3305 \pm 83$ events for $10,29.5$, and $67 \mathrm{~Hz} \mathrm{BF}$, respectively. This is more than one order of magnitude slower than previously described forms of depression at PC_DCNs (Telgkamp and Raman, 2002; Pedroarena and Schwarz, 2003; Turecek et al., 2016), including a "slow" frequency-dependent depression (i.e., with time constant of 117 events for $100 \mathrm{~Hz}$ stimulus trains) found in smaller DCNs $(15-20 \mu \mathrm{m})$ from younger animals (P13-P15), recorded at lower temperatures $\left(31^{\circ} \mathrm{C}\right)$ (Telgkamp and Raman, 2002). Significant differences in average IPSC amplitudes as a function of the BFs could be detected considering events 1000-1100 or further (Fig. 1G, bottom). Moreover, the results from single experiments agreed with the population ones (Fig. $1 H$ ): in all but 1 case, the mean IPSCs amplitude decreased with increasing BFs $(0.402 \pm 0.02$ vs $0.29 \pm 0.02, n=8$, paired $t$ test, $p<0.001$ for 10 vs $29.5 \mathrm{~Hz}$; and $0.29 \pm 0.02$ vs $0.24 \pm 0.02$, for $29.5 \mathrm{~Hz}$ vs $67 \mathrm{~Hz}, n=9$, paired $t$ test, $p=0.003$ ).

Similar results arose using Poisson-like trains of stimuli with mean frequencies 10 or $70 \mathrm{~Hz}$ (Fig. $2 \mathrm{~A}$ ), ruling out that the alternation of regular and irregular periods of firing in awake animals (Shin et al., 2007; Hong et al., 2016) could result in recovery from depression during long intervals abolishing the frequency differences in synaptic strength found here. Importantly, despite developmentally regulated PC_DCN STP (Turecek et al., 2016, 2017), I found similar late phase of slow frequency-dependent decay in IPSC amplitude along trains using slices from adult mice (P55-P72), albeit with shallower levels of depression at steady state than at younger ages (Fig. $2 B$ ). In addition, similar to previous studies using slices from mature animals $(>\mathrm{P} 20)$, recordings from large DCNs, and close to physiological temperatures (Najac and Raman, 2015; Turecek et al., 2016) $\left(33^{\circ} \mathrm{C}-34^{\circ} \mathrm{C}\right.$ and $34^{\circ} \mathrm{C}-35^{\circ} \mathrm{C}$, respectively), PC_DCN activation did not evoke prominent slow tonic currents, in contrast to recordings using smaller DCNs $(15-20 \mu \mathrm{m})$, from younger mice (P13-P15), and lower recording temperatures $\left(31^{\circ} \mathrm{C}\right)$ (Telgkamp and Raman, 2002). Type of cell, developmental stage, and temperature all influence PC-DCN kinetics and could explain differences in slow currents (Linnemann et al., 2004; Person and Raman, 2012; Najac and Raman, 2015; Turecek et al., 2016).

Together, these results strongly argue that regular or irregular sustained activity of young or adult PC_DCNs induces a previously undetected slow form of frequency-dependent STD (SSTD), requiring hundreds to thousands of events to reach steady-state level.

Because of the acknowledged difficulties in carrying large DCN recordings using slices from older animals (Uusisaari et al., 2007; Turecek et al., 2016) and the need of particularly long recordings for the present study, the rest of the experiments were performed using slices from juvenile mice ( $>$ P20) as in other studies (Turecek et al., 2016, 2017).
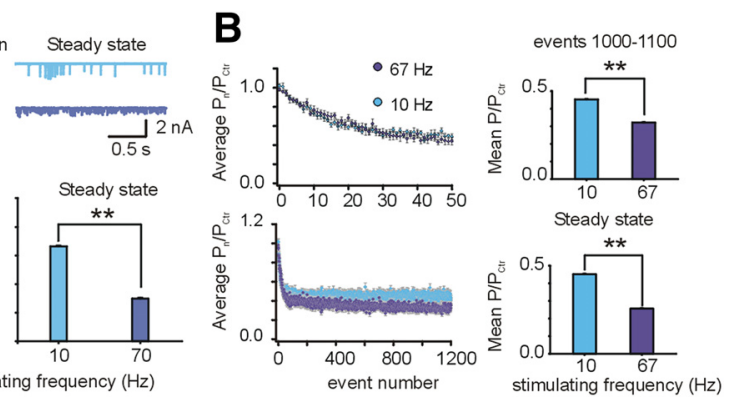

stimulating frequency $(\mathrm{Hz})$

Figure 2. S-STD frequency dependence under irregular activation patterns and in DCNs from adult mice. $A$, Top, 作 列 Figure 1, but for Poisson-like stimulation at $10 \mathrm{~Hz}(n=9)$ and $70 \mathrm{~Hz}(n=7)$. Significance assessed using Mann-Whitney rank sum test $(0.33 \pm 0.002,0.23 \pm 0.003$ for 10 and $70 \mathrm{~Hz}$, for events 1000-1100th; and 0.33 $\pm 0.002,0.15 \pm 0.003$ for 10 and (1000-1100th for 10 and $67 \mathrm{~Hz}$ BFs: $0.45 \pm 0.002$ and $0.32 \pm 0.002, t$ test, $p<0.001$; average of 100 events at steady state: $0.45 \pm 0.002$ and $0.26 \pm 0.003$ for 10 and $67 \mathrm{~Hz}$, respectively, $t$ test, $p<0.001$ ). ${ }^{* *} p<0.001$.

\section{Sustained PC activation modulates IPSCs and DCN spike responses evoked by HFS trains}

Given the typical PCs sustained activity found in awake-behaving animals (e.g., Schonewille et al., 2006), presumably S-STD continuously controls PC_DCN efficacy. This raises the question of whether and how S-STD modulates the responses to more transient changes in PC rate associated to cerebellar controlled behaviors. To address this question experimentally, first, PC_DCN synapses were depressed to steady state using protracted stimulation at different BFs; and afterward, a brief, high frequency train of stimuli (HFS, 100 events, $180 \mathrm{~Hz}$ ) was used to mimic behavior-driven transient signals. The HFS duration covers the length of usual discrete motor events (e.g., reaches, steps, saccades, etc.). To quantify and compare the synaptic output induced by HFSs preceded by different BFs, I measured the total charge transferred during the HFS $\left(Q_{H F S}\right.$, see Materials and Methods), then normalized it to the charge transferred by corresponding control IPSCs $\left(\mathrm{Q} \mathrm{P}_{\mathrm{Ctrl}}\right)$ to be able to average across neurons, and expressed it per second. Analysis of changes in $\mathrm{Q}_{\mathrm{HFS}}$ as a function of the preceding BF revealed a remarkable modulation (Fig. 3A). Specifically, the normalized QHFS decreased with increasing BF (no prior activity, $0 \mathrm{~Hz}$ : $72 \pm 4.5, n=$ 22; BF $10 \mathrm{~Hz}: 61 \pm 3.3, n=16,29.5 \mathrm{~Hz}: 40 \pm 4.0, n=9$, and $67 \mathrm{~Hz}: 16 \pm 1.9, n=12, t$ test: 0 vs $10 \mathrm{~Hz}, p=0.033,1$ tail; 10 vs $29.5 \mathrm{~Hz} ; p<0.001 ; 29.5$ vs $67 \mathrm{~Hz}, p<0.001)$. These $\mathrm{Q}_{\mathrm{HFS}}$ values contrast with the expected for either, a nondepressing synapse (considering 1 the charge transfer of control IPSCs and 180 the HFS frequency: $1 \times 180=180$ ), or for synapses that after the first phase of depression remain stable and frequency-invariant (Fig. $1, \sim 0.48 \times 180=86$ ). The mean $\mathrm{Q}_{\mathrm{HFS}}$ for HFSs preceded by $2 \mathrm{~min}$ stimulation at $67 \mathrm{~Hz}$ was approximately one-fourth of the $\mathrm{Q}_{\mathrm{HFS}}$ elicited by HFSs preceded by $10 \mathrm{~Hz}$ stimulation. These results strongly support the view that BFs modulates the output produced by behavior-driven PC signals.

To further probe the latter idea, I used cell-attached recordings of spontaneously firing DCNs and similar stimulating protocols as in Figure $3 A$, to investigate whether the changes in synaptic output translated to changes in DCN spiking activity (Fig. $3 C, D$ ). Large mature DCNs recorded in vitro at physiological temperatures fired spontaneously at high frequencies (mean firing rate $81.9 \pm 3.5 \mathrm{~Hz}$, range $48-121 \mathrm{~Hz}, n=12$ ), which are 
A Whole cell DCN recordings

$2 \mathrm{~min}$ BF Stim. $\rightarrow \mathrm{HFS}(180 \mathrm{~Hz})$

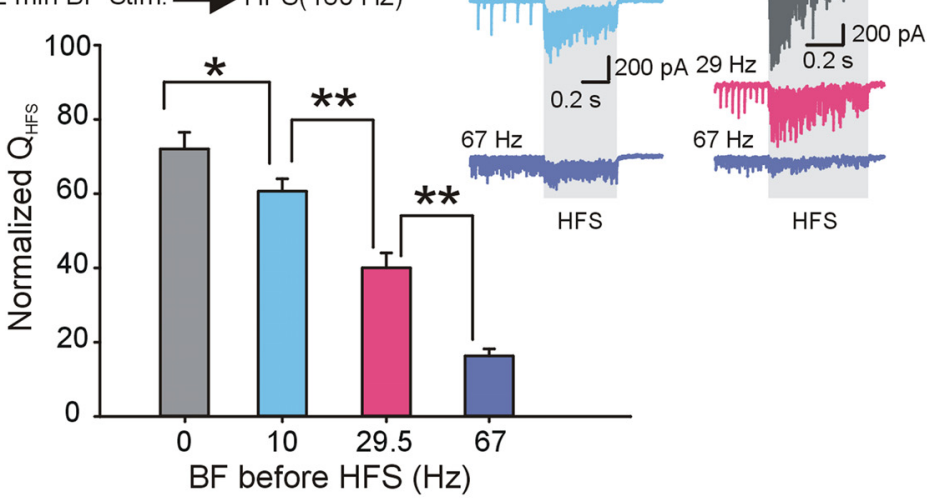

c

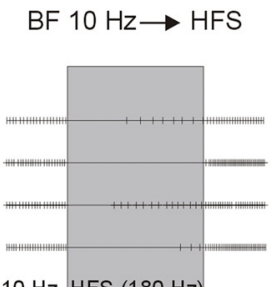

$10 \mathrm{~Hz}$ HFS $(180 \mathrm{~Hz})$

E

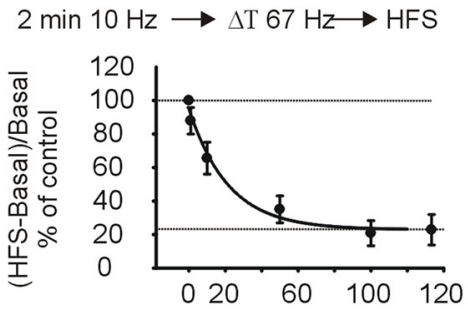

Duration $67 \mathrm{~Hz}$ stimulation (s)
B $180 \mathrm{~Hz}$

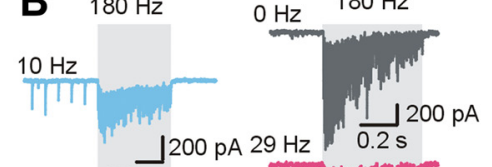

results were similar to those using $120 \mathrm{~s}$ of sustained stimulation indicating that at steady state BF determined S-STD. Thus, the results were pooled together for statistical analysis. Individual and summary results show that HFSs preceded by sustained stimulation at $10 \mathrm{~Hz}$ completely suppressed or sharply decreased DCN firing rate, while HFSs delivered after $67 \mathrm{~Hz}$ only induced a moderate effect (Fig. $3 C$, D) $(-67.7 \pm 7.4$ and $-26.5 \pm 6.6 \mathrm{~Hz}$, respectively, paired $t$ test, $p<0.001, n=$ $12)$. These results strongly suggest that the modulation induced by S-STD not only has an impact on the synaptic output but on DCN firing rate, and thus possibly on the cerebellar output in behaving animals.

Having found that S-STD modulates DCN responses to HFSs, I next explored the time course of this effect. This question is relevant because (1) it could provide information on the existence and duration of a time window for linear encoding; and (2) it could inform on the consequences of changes in PC background activity in different timescales. To explore the time course, I leveraged the above-described differential modulation of HFS spiking responses according to preceding $\mathrm{BFs}$ (Fig. 3C,D). Using cell-attached recordings, as above, I interposed between the 2 min blocks of stimulation at 10 or $67 \mathrm{~Hz}$ and the HFSs, a switch in the stimulating frequency (to 67 or $10 \mathrm{~Hz}$, respectively), for variable periods of time $(\Delta \mathrm{T}$, shown $1,10,50$, or $100 \mathrm{~s}$; Fig. $3 E, F$, top). The change in DCN firing rate for the 10 to $67 \mathrm{~Hz}$ switches (normalized to the response to HFSs preceded by $120 \mathrm{~s}$ at $10 \mathrm{~Hz}$ without switch to $67 \mathrm{~Hz}$ ) decreased with increasing $\Delta \mathrm{T}$ at $67 \mathrm{~Hz}$, with a time constant of $22 \mathrm{~s}$. The change was undetectable for $67 \mathrm{~Hz}$ periods of $\leq 1$ $s$ and, on average, only after $100 \mathrm{~s}$ the suppression reached the same level than after 2 min of stimulation at $67 \mathrm{~Hz}$. For the switch from 67 to $10 \mathrm{~Hz}$, the time constant of recovery was $14 \mathrm{~s}$ (i.e., the recovery from deeper depression seemed faster than the buildup), but within a similar time range, and it took also similar periods to reach a new steady-state level.

within the DCN firing rate range found in behaving mice at rest (e.g., Ten Brinke et al., 2017; Sarnaik and Raman, 2018). Furthermore, under cell-attached recordings, the intracellular content is not dialyzed, reflecting the conditions found in the intact animal/neuron. In a number of experiments, I used the same number of preceding stimuli $(7000$ stimuli at 10 or $67 \mathrm{~Hz})$ to rule out that the number of stimuli (and not the stimulating frequency) determined the changes in synaptic strength. The
Again, no changes were detected for periods of $\leq 1 \mathrm{~s}$ (Fig. $3 F$ ). The bidirectional amplitude modulation established in the course of tens of seconds highlight the time scale and flexibility of S-STD and consequent HFS response modulation. Moreover, the slow time course (from tens of seconds to minutes) contrasts with the rapid adaptation (in the millisecond range) of other forms of depression expressed earlier during the train (Telgkamp 
and Raman, 2002), strongly suggesting that S-STD is based on a different mechanism. The minor differences in buildup and recovery time course of S-STD could depend on differences in the activation or deactivation rates of faster synaptic forms of plasticity and/or S-STD itself.

Importantly, these results revealed a time window up to few seconds during which changes in $\mathrm{BF}$ did not significantly alter the response to subsequent HFS; in other words, the PC_DCN synaptic gain seemed unchanged over this period. Remarkably, this is the range of durations of common motor events controlled by cerebellum. If S-STD guaranteed stable synaptic gain during this time window for all functionally relevant PC rates, then S-STD could support linear encoding of behavior-related PC signals while still being able to adapt synaptic strength to the background activity.

\section{Linear input/output transfer} function for 0.5-s-long PC test trains with gain dependent on PC BF

To test the above-mentioned idea, I used $500 \mathrm{~ms}$ test trains of stimuli, with frequencies ranging between 10 and $300 \mathrm{~Hz}$ to match the usual PC signals related to common movements, preceded by protracted PC activation with different BFs (Fig. 4A, top). To estimate the synaptic output evoked by the test trains, I measured the charge transfer amplitude as in Figure 3A, B (Q test, normalized to Q $\mathrm{P}_{\mathrm{Ctrl}}$, and expressed per time unit; for further details, see Materials and Methods), and plotted it against the corresponding test train frequency for each explored BF (Fig. 4A). Indeed, in favor of the idea that $\mathrm{S}$ STD provides a time window for linear encoding, the $\mathrm{Q}$ test values were proportional to the corresponding test train frequency, such that the relationship between these two parameters was approximately linear over the physiological range of PC rates $(10-200 \mathrm{~Hz})$, with slopes or gains decreasing for increasing BFs. In contrast, the transfer function corresponding to test trains applied from rest displayed a saturating type of curve characteristic of depressing synapses $(0 \mathrm{~Hz}$, black empty circles). The dashed unity line indicates the
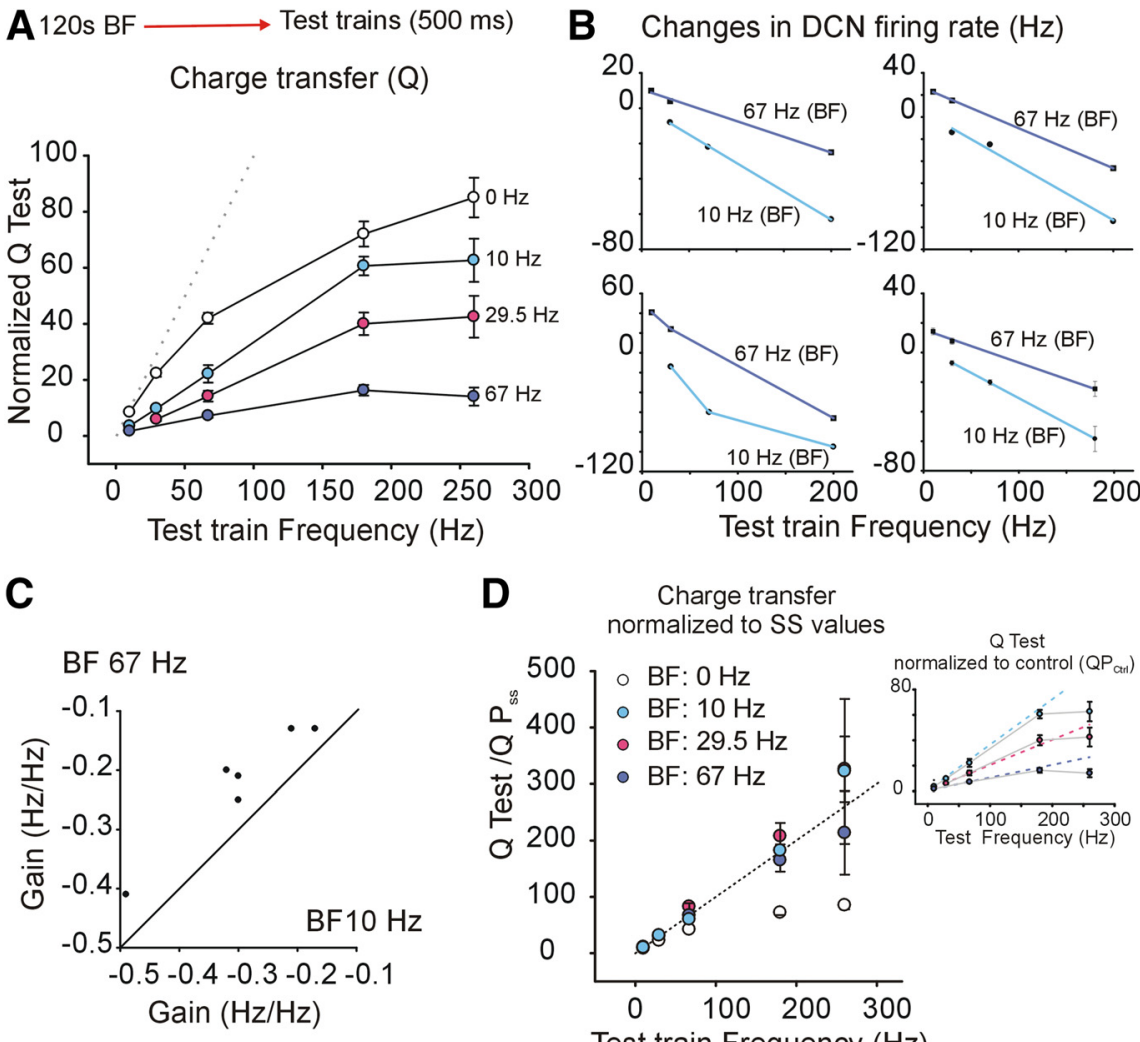

D
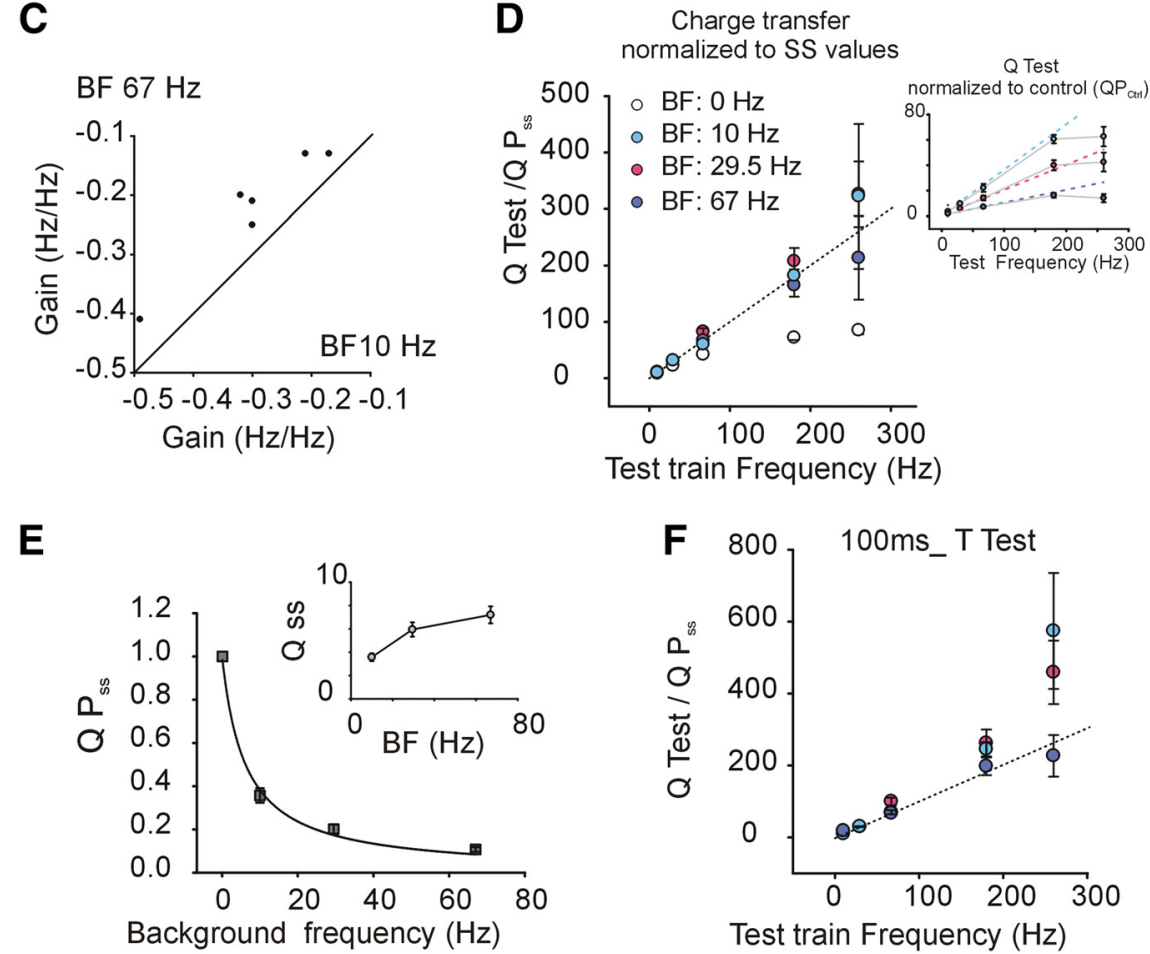

Figure 4. Linear PC_DCN transfer function of subsecond changes in PC rate with gain dependent on preceding BF. $\boldsymbol{A}$, Top, Stimulation paradigm to investigate changes in PC_DCN input/output gain: $0.5 \mathrm{~s}$ test trains of stimuli at different frequencies (from 10 to $260 \mathrm{~Hz})$ delivered after $120 \mathrm{~s}$ of stimulation at different BFs $(0,10,29.5$, or $67 \mathrm{~Hz})$. Bottom, Summary of measured PC_DCN synaptic charge transfer during the test trains $\left(Q_{\text {TEST }}\right)$, normalized to the charge transfer of corresponding control IPSCS $\left(Q P P_{\text {trr }}\right)$, expressed per time unit, as a function of the test frequency. The plots corresponding to test trains applied from rest $(0 \mathrm{~Hz})$, or after $120 \mathrm{~s}$ at 10,29.5, or $67 \mathrm{~Hz}$ are labeled on the right of each curve (color code as before). Note the almost linear relationship between 10 and $180 \mathrm{~Hz}$ and the different slopes for different BFs, indicative of different gains (gain of linear fits from 10 to $200 \mathrm{~Hz}$ : $0.34 \pm 0.002,0.23 \pm 0.001$, and $0.085 \pm 0.004$ for $10,29.5$, and $67 \mathrm{~Hz}$, respectively). Dashed black line (unity line) indicates the expected values for nondepressing/nonfacilitating PSCS. B, Plots of changes in DCN firing rate (difference to steady state rate) as a function of the test train frequency from experiments using the same stimulation paradigm as in $\boldsymbol{A}$, and DCN cell-attached recordings. Top, Two typical examples exhibit approximately linear relationships (lines correspond to linear fits to the data). Left, bottom, One unit exhibited nonlinear relationship after $10 \mathrm{~Hz}$ BF (points linked by straight lines). Right, bottom, Average changes in DCN firing rate from neurons with linear relationship $(n=6$, lines correspond to linear fits; gain: $-0.34 \pm 0.005$ and $-0.22 \pm 0.012 \mathrm{~Hz}$ / $\mathrm{Hz}$ and $R^{2}=0.999$ and 0.997 for 10 and $67 \mathrm{~Hz}$ BF, respectively). C, Scatter plots of gains (estimated by the slopes of linear fits as in $\boldsymbol{B}$ ) for $\mathrm{BF} 67 \mathrm{~Hz}$ versus their corresponding values for $\mathrm{BF} 10 \mathrm{~Hz}(n=6)$ for single neurons (Wilcoxon sign rank test, $p=0.031$, $n=6$; diagonal is the unity line). $\boldsymbol{D}$, Same data from $\boldsymbol{A}$, but normalized to the charge transferred by the corresponding IPSCS at steady state $\left(Q P_{S S}\right)$. The responses to test stimuli applied from rest, $0 \mathrm{~Hz}$, in empty black. The plots corresponding to different BFs overlap around the unity line indicating that the synaptic strength at steady state determines the BF-dependent gains (i.e., the slopes shown in $A$ ). Linear fits for the test frequency range $10-200 \mathrm{~Hz}$ (for clarity not shown). For BF $10 \mathrm{~Hz}$ : slope $1.0 \pm 0.04, R^{2}=$ 0.99; BF $29.5 \mathrm{~Hz}$ : slope $1.1 \pm 0.06, R^{2}=0.99 ; \mathrm{BF} 67 \mathrm{~Hz}$ : slope $0.9 \pm 0.03, R^{2}=0.99$. Dashed line indicates unity line. Inset, same plot as in A overlaid to colored dashed lines indicating the result of multiplying the corresponding mean $Q P_{s s}$ by the test train frequency, for each BF. $E$, Average charge transfer amplitude for IPSCs at steady state normalized to control IPSCS ( $\left.P_{s s}\right)$ as a function of the $\mathrm{BF}(n=25)$. Curve indicates the fit with a rational function $\left(n=25, R^{2}=0.999\right)$. Inset, Averaged measured charge transfer amplitude at steady state per time unit, normalized to control IPSCs ( $Q$ ss) versus the BF (note different scale). $\boldsymbol{F}$, Same as in $\boldsymbol{D}$, but for the first $100 \mathrm{~ms}$ of the responses to the 500 ms test trains show gain $>1$. 
expected values for a nondepressing/nonfacilitating synapse. These results strongly support the view that S-STD provides a time window of hundreds of milliseconds to seconds over which PC rates can be linearly encoded in the synaptic output.

To assess the functional relevance of these results, a different series of experiments using DCN cell-attached recordings tested whether similar stimulation patterns as in Figure $4 A$ induced equivalent changes in DCN firing rates (Fig. $4 B, C$ ). Although synchronous PC inputs are expected to be less effective than desynchronized ones in changing DCN firing rates (Sarnaik and Raman, 2018), 6 of 7 recorded DCNs (average basal rates preceding stimulation $83.7 \pm 4.8 \mathrm{~Hz}$, range $60-121 \mathrm{~Hz}, n=7$ ) displayed linear decreases in firing rates with increasing test frequencies (for examples, see Fig. 4B, top). Moreover, the gain of these relationships, that is, the decrease in DCN firing rate per increase in $\mathrm{PC}$ test frequency was in each case higher for test trains preceded by $10 \mathrm{~Hz}$ BF compared with those applied after $67 \mathrm{~Hz}$ (Fig. $4 A$, bottom right, $C$ ). Thus, these results strongly suggest that S-STD is able to support scaled linear encoding of PC rates in the DCN spike output, over behavior-relevant time windows. These results do not rule out that under different conditions (i.e., responsive state of DCNs, presence of other inputs, or other PC input patterns), DCNs could respond with other spike outputs, a matter for further studies but indicate that DCN spike output suppression can faithfully reflect PCs inhibitory drive, as suggested previously (Pedroarena, 2010).

The finding that the gain of PC_DCN transfer function depends on the preceding BF opens two types of questions: (1) about the origin and (2) about the consequences of BF-dependent gains. Regarding the first, different gains could derive simply from the differences in synaptic strength caused by S-STD. Alternatively or additionally, if S-STD involved modifications in fast forms of STP (e.g., decreased facilitation), different balance of fast facilitation/depression in response to the test trains could contribute to the different gains. To elucidate this issue, I renormalized the data in Figure $4 A$ to the charge transferred by the corresponding IPSCs at steady state $\left(\mathrm{Q}_{\mathrm{ss}}\right.$; Fig. $\left.4 D\right)$. After this transformation, over the range of $10-200 \mathrm{~Hz}$, the gain of the curves corresponding to different BFs collapsed, coinciding with the unity line, consistent with the notion that the different gains were exclusively dependent on the preceding level of synaptic strength. This is further illustrated in the inset, where the experimentally obtained $Q_{\text {test }}$ values (plotted as in Fig. $4 A$ ) are similar to the values calculated by multiplying the average $\mathrm{Q} \mathrm{P}_{\text {ss }}$ for different BFs (see later), and the test train frequencies (dashed lines, color code as before). Together with recent reports of linear transfer function of PC_DCN synapses after tens of events, when S-STD is not yet expressed (Turecek et al., 2016, 2017), this outcome strongly suggests that S-STD expression did not interfere with the mechanism(s) responsible for linear encoding, which could be the equilibrated fast facilitation and depression.

Regarding the question of the consequences of BF-dependent gain for PC-DCN function, it is important to note, first, that because the synaptic gain at steady state determines the gain for the test train periods, the gains are the same when considering changes from steady state and, thus, effective under the typical incessant PC activity. Inspection of the results in Figure $4 \mathrm{~A}$ reveals one possible consequence of BF-dependent gain. A jump to $180 \mathrm{~Hz}$ from $\mathrm{BF} 10 \mathrm{~Hz}$ resulted in a change in synaptic output of 57 charge units, in contrast to the 9.1 units produced from BF $67 \mathrm{~Hz}$. Thus, changes in background rate could represent a way for tuning the responses of different PCs to stereotyped signals (e.g., complex spikes). Further inspection of the same results illustrates another possible consequence. It has been previously shown that for many fast depressing synapses, changes in gain determine a Weber-Fechner effect (i.e., independently of the BF), equal fractional changes in frequency $(\Delta \mathrm{F} / \mathrm{BF})$ produce equal absolute outputs, despite the difference in absolute presynaptic rate change (Abbott et al., 1997). Figure $4 A$ shows that here, similarly, a change from BF $10-29.5 \mathrm{~Hz}(\sim 20 \mathrm{~Hz}, 200 \%$ of change) produced a change in output of 6.2 charge units, which is close to the 9.1 units resulting from changing BF 67 to $180 \mathrm{~Hz}$ ( $113 \mathrm{~Hz}$, but $\sim 170 \%$ of change). For many fast depressing synapses, synaptic strength at steady state becomes proportional to $1 /$ BF with increasing BFs (Abbott et al., 1997; Tsodyks and Markram, 1997), such that the response to a change in frequency becomes transiently proportional to $\triangle \mathrm{F} / \mathrm{BF}$, explaining the Weber-Fechner effect. For PC_DCNs, the charge transfer produced by single IPSCs at steady state $\left(\mathrm{Q}_{\mathrm{ss}}\right)$ plotted as a function of the BF shows $\mathrm{Q} \mathrm{P}_{\mathrm{ss}}$ decreasing nonlinearly in proportion to 1/ $\mathrm{BF}$ with increasing BFs (Fig. $4 E$, black line: a rational function fit). This finding suggests S-STD, too, could support a WeberFechner effect, but importantly, for time windows in the timescale of usual movements. Indeed, calculated from the data in Figure $4 A$, the changes in synaptic charge transfer as a function of $\Delta \mathrm{F} / \mathrm{BF}$ were similar for $\mathrm{BFs}>10 \mathrm{~Hz}(6.69 \pm 0.05$ and $5.69 \pm 0.26$ charge units, for $\mathrm{BF} 29.5$ and $67 \mathrm{~Hz}$, respectively). Thus, S-STD could support similar impact on target DCNs from PCs with different BFs.

A different consequence of the BF-dependent gain is that, as indicated in the inset of Figure $4 E$, the charge transferred at steady state per time unit (Q SS) increases nonlinearly with the BF (compare with Fig. $4 A$ ), signaling low sensitivity of PC_DCNs to BFs. This property could be useful to prevent silencing of DCNs with increasing BFs and in general to maintain DCNs within their working range.

Overall, these results indicate the following: (1) the BF-dependent gain modulation reduces the sensitivity to steady PC rates; (2) PCs independently of their BF would produce equal outputs to equal fractional changes in rate; and (3) changes in PC BF could be used to tune the response to stereotyped signals (e.g., complex spikes).

Finally, the observation that changes in PC stimulation frequency often produced relative transient facilitation of IPSCs (e.g., Fig. 3B) (Pedroarena and Schwarz, 2003; Turecek et al., 2016, 2017), and that some cerebellar signals are short-lasting, raised the question of whether the linear transfer holds for signals shorter than the test trains used here. I analyzed the charge transferred during the first $100 \mathrm{~ms}$ of the test trains when relative facilitation is predominant (Fig. $4 F$; Q, test normalized to $\mathrm{Q} \mathrm{P}_{\mathrm{ss}}$ as in Fig. 4D). The transfer function for the $100 \mathrm{~ms}$ periods was approximately linear within the $10-200 \mathrm{~Hz}$ range but with slopes slightly $>1$, suggesting linear and preferential transfer of brief signals or the onset of longer ones. In addition, for the higher test frequencies $(260 \mathrm{~Hz})$, the plots deviated from linearity depending on the preceding BF: after 10 or $29.5 \mathrm{~Hz}$, the responses were often supralinear, although variable (note the high SEM). The variability is in line with the idea that similar type of synapses differ mainly by the degree of facilitation (Fekete et al., 2019), but other mechanisms cannot be ruled out and should be addressed in further studies. These results unveil a new role of facilitation at PC_DCN synapses in promoting the transfer of short signals, in addition to its possible role in linear encoding.

Together, these findings strongly suggest that, in the timescales of usual motor behaviors, S-STD enables scaled linear 
A
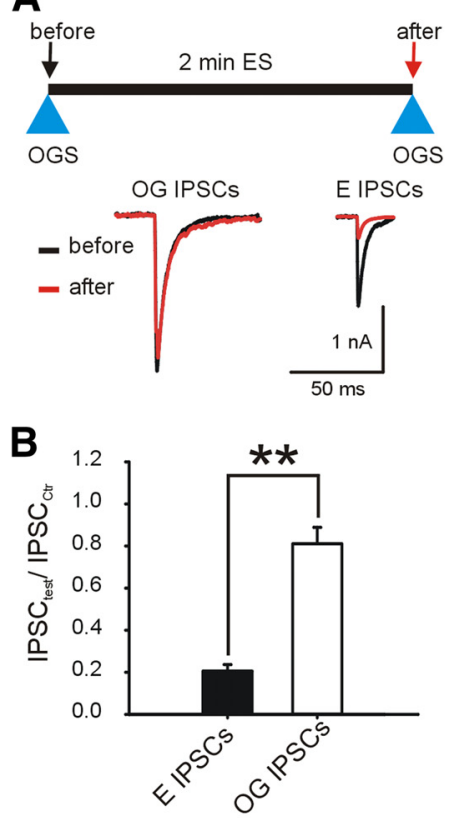

E
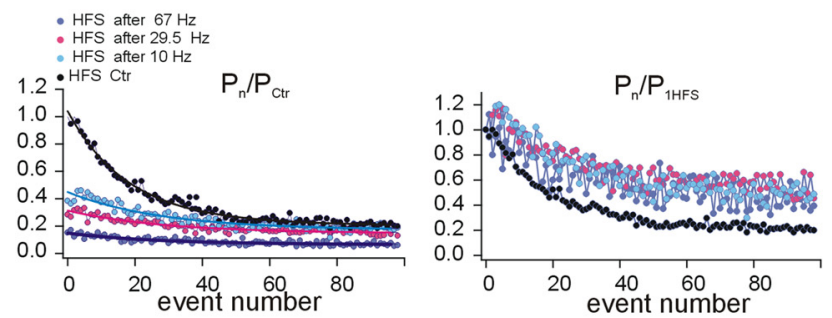

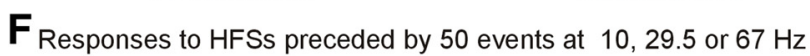
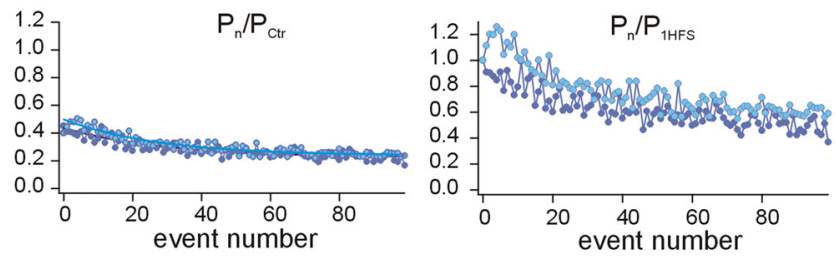

Responses to HFSs preceded by 50 evs. or 120 s Stim.
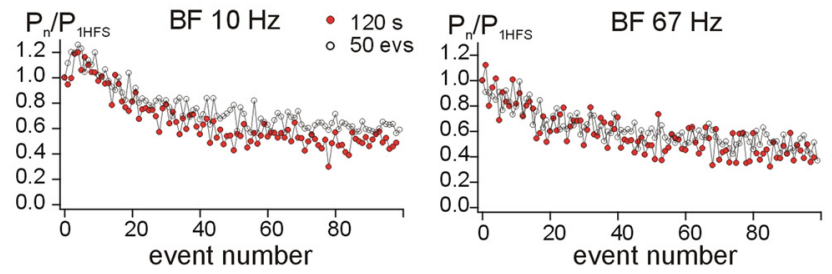

Figure 5. Exploration of S-STD mechanism. A, Combined OG and ES to explore input specificity. Top, Experimental paradigm. Bottom, Representative examples of OG IPSCS and ES IPSCs before (control, black) and after (test, red) 2 min ES at $67 \mathrm{~Hz}$. B, Plot showing significant differences in average $O G$ and ES IPSC amplitudes after 2 min ES at $67 \mathrm{~Hz}$ (normalized to the corresponding controls: IPSCs induced after 2 min without ES; $0.81 \pm 0.078$ and $0.21 \pm 0.03$ for $0 G$ and ES IPSCs, respectively, paired $t$ test, $p<0.001, n=8$ ) suggests input specificity. C, Plot of $\mathrm{CV}^{-2}$ versus their corresponding mean amplitudes $(M)$ for IPSCS depressed to steady state steady using $120 \mathrm{~s}$ stimulation at $10,29.5$, or $67 \mathrm{~Hz}$, normalized to the values of their corresponding control IPSCS $(n=8)$. Inset, Expected $\mathrm{CV}^{-2}$ versus $M$ values for changes in the parameters $N$ (red line), $p$ (green), or $q$ (blue) of a binomial release model. D, Same as in C, but for IPSCs depressed using BF 29.5 or $67 \mathrm{~Hz}$ normalized the corresponding $\mathrm{BF} 10 \mathrm{~Hz}$ values $(n=7)$. Dashed line indicates unity line. Fit of data using a linear function $\left(R^{2}=0.78\right.$, slope $1.07 \pm 0.13, n=7$; data not shown). $\boldsymbol{E}$, Left, Plot of mean IPSC peak amplitude normalized to control IPSCS as a function of the HFS event number for HFSs (100 events, $180 \mathrm{~Hz}$ ) applied from rest (black, $n=17$ ) or after $120 \mathrm{~s}$ of stimulation at $10 \mathrm{~Hz}$ (light encoding of PC rates in both the synaptic output and the DCN rates while producing adaptation at longer timescales.

Evidence that S-STD mechanism is input-specific, presynaptic, and independent of fast forms of plasticity

To understand the basis of the apparently opposing S-STD effects at different timescales, first, I set out to explore the S-STD mechanism. S-STD input specificity was investigated by combining OG and ES in slices from mice expressing channelrhodopsin in PCs under the L7 promoter (L7-ChR2-eYFP; for further details, see Materials and Methods) (Chaumont et al., 2013). I adjusted the stimulation intensities to obtain OG IPSCs larger than the ES IPSCs under control conditions and assumed this reflects a larger number of OG activated synapses. If depression was input-specific, tonic ES should depress transmission at ESactivated synapses but spare those exclusively (or mainly) activated by light. Indeed, after $2 \mathrm{~min}$ of repetitive ES at $67 \mathrm{~Hz}$, the depression of OG IPSCs was significantly less than for ES IPSCs, supporting the idea that depression is input-specific (Fig. 5A,B). The much lower depression of OG IPSCs suggested that the OG and ES stimuli were largely nonoverlapping. This is likely, since OG activation was submaximal (see Materials and Methods). The possibility that OG activation modified synaptic function (e.g., by inducing prolonged depolarization of terminals and branches or calcium influx promoting alternative release pathways) could not be completely ruled out. However, in one experiment where the degree of occlusion of OG and ES IPSCs was monitored, the degree of depression of the OG IPSC coincided with the expected depression of the overlapping component (not shown), supporting the idea that the results reflected S-STD input specificity.

Another important conclusion drawn from the small changes induced by 120 s ES in OG IPSCs compared with ES IPSCs is that changes in chloride gradient is unlikely the cause for S-STD (as OG IPSCs recorded from the same DCNs would have been expected to change if this were the case).

To gain further insight into the basis of S-STD, I investigated changes in the inverse squared $\mathrm{CV}^{-2}$ of IPSCs, an index of synaptic variability useful to clarify the mechanism underlying changes in synaptic efficacy (Bekkers and Stevens, 1990; Malinow and Tsien, 1990) (for details, see Materials and Methods). Briefly, specific changes in $\mathrm{CV}^{-2}$ versus the respective changes in $\mathrm{M}$ are expected from changes in each one of the factors assumed to explain the mean IPSC amplitude $(M=$ N.p.q), that is, the number of presynaptic releasable vesicles $(N)$, the

blue, $n=11$ ), $29.5 \mathrm{~Hz}$ (red, $n=9$ ), and $67 \mathrm{~Hz}$ (dark blue, $n=9$ ). Continuous lines indicate exponential fits to the curves. Note the faster decay for the HFS applied from rest but similar slower values for the other curves, suggesting similar probability of release after the first events. Right, Plot of IPSC peak amplitudes normalized to the value of the first IPSC of the HFS, illustrates relative facilitation during the first events for $\mathrm{BFs} 10$ and $30 \mathrm{~Hz}$, but similar decay rate and percentage of decay for different BFs afterward. $\boldsymbol{F}$, Left, Same as in $\boldsymbol{E}$ (left), but for HFSs applied after 50 events at 10 or $67 \mathrm{~Hz}$ (color code as in $\boldsymbol{E}$ ). Note the relative facilitation of the first events for $\mathrm{BF} 10 \mathrm{~Hz}$, but similar degree of depression at the end of the HFS for 10 and $67 \mathrm{~Hz}$ BF. Continuous lines indicate exponential fits. The data correspond to a different set of neurons than those shown in $\boldsymbol{E}$. Right, Same data as on the left, but normalized to the corresponding first IPSC of the HFS. Bottom, Plots represent superimposed mean IPSC amplitude normalized to the first HFS event for HFSs delivered after $120 \mathrm{~s}$ of stimulation (red circles) or 50 events (empty circles) after BF $10 \mathrm{~Hz}$ (left) or $67 \mathrm{~Hz}$ (right) as a function of the HFS event number. The similarity in the IPSC amplitude time course after $120 \mathrm{~s}$ or 50 events suggests that S-STD did not alter the relative proportion of fast synaptic facilitation and depression evoked by the HFSs. ${ }^{* *} p<0.001$. 
probability of each one to be released $(p)$, and the postsynaptic response to one vesicle $(q)$ (for guidance, see Fig. 5C, inset). Figure $5 \mathrm{C}$ illustrates how the $\mathrm{CV}^{-2}$ of PC_DCNs IPSCs depressed to steady state (normalized to control IPSCs) decreased more than the corresponding means. This result is compatible (1) with a presynaptic locus because changes in $q$ would have caused no change in $\mathrm{CV}^{-2}$; and (2) with a reduction in $p$, because changes in $N$ would have caused proportional changes in the $\mathrm{CV}^{-2}$ and $M$ (see inset). Next, to explore the specific basis of S-STD, I compared IPSCs depressed with steady state using different BFs (Fig. 5D). The $\mathrm{CV}^{-2}$ of IPSCs evoked by BF 29.5 or $67 \mathrm{~Hz}$ normalized to the values corresponding to $\mathrm{BF}$ $10 \mathrm{~Hz}$ decreased proportionally to their means, a result consistent with a presynaptic mechanism and particularly with a change in the number of available quanta $(N)$. Overall, this analysis suggests that repetitive stimulation from rest induces first a decrease in the probability of release and that S-STD depends on a presynaptic mechanism, specifically, a decrease in the available quanta $(N)$. Moreover, the present results are incompatible with S-STD being dependent on changes in $q$, for example, due to changes in the postsynaptic chloride gradient by sustained stimulation or in the amount of NT contained in each quantum (Fig. 5C, inset, blue line).

To verify the $\mathrm{CV}^{-2}$ analysis outcome, an alternative estimation of the release probability was obtained. The rate with which the peak amplitude of synaptic responses decay during a train is in part dependent on the release probability, as larger use of the available quanta would deplete faster the remaining pool (Buchs and Senn, 2002). Because S-STD adjusts slowly (Fig. 3), one would expect persistent changes in the release probability if this were the basis for S-STD. Thus, I analyzed the rate of decay of peak amplitudes of IPSCs evoked by HFSs $(180 \mathrm{~Hz})$ applied from rest or after different periods of protracted PC stimulation. For HFSs delivered after $120 \mathrm{~s}$ at different BFs, the IPSC peak amplitudes decayed to different steady-state levels depending of the preceding BF (Fig. $5 E$ ), in agreement with the idea that S-STD adjust slowly to new levels (see later for details). The rate of decay for HFSs preceded by $120 \mathrm{~s}$ stimulation at different BFs was similar but slower than for HFSs applied from rest (Fig. 5E; exponential fits to the data, tau: $18.6 \pm 0.53,27.9 \pm 3.1,28.7 \pm 3.6$, and $27.7 \pm 5.1$ for rest $[n=34]$, and BFs $10[n=11], 29.5[n=10]$, and $67[n=9] \mathrm{Hz}$, respectively). This is consistent with a decrease in release probability at the beginning of the train but not between different S-STD levels of depression. Moreover, normalizing the amplitudes to the first IPSC of the HFS more clearly illustrates the similarity in decay rate and proportion of decay for HFSs preceded by different BFs (Fig. $5 E$, right). In contrast, in a different set of experiments using HFSs applied after 50 events at 10 or $67 \mathrm{~Hz}$, the IPSC peak amplitude decayed to similar steadystate levels (Fig. 5F; IPSC amplitude averaged over the last 30 events, normalized to control IPSC: $0.247 \pm 0.079$ and $0.23 \pm$ 0.07 for 10 or $67 \mathrm{~Hz}$, respectively, paired $t$ test, $n=5, p=0.162$ ). These results demonstrate that the synaptic strength can be rapidly adjusted to new levels before S_STD expression (Telgkamp and Raman, 2002) (compare with Fig. 5E, mean IPSC peak amplitude over the last HFS 30 events after 2 min stimulation: $0.185 \pm 0.25$ vs $0.067 \pm 0.09$ for 10 or $67 \mathrm{~Hz} \mathrm{BF}$, respectively, $n=11$ and $n=9$, Mann-Whitney rank sum test, $p=0.001)$. The rates of decay for HFS after 50 events at different BFs were similar (tau: $27.8 \pm 3$ and $31.2 \pm 5.1$ events for BF $10[n=5]$ and 67 $[n=5] \mathrm{Hz}$, respectively), and similar to rates of decay for HFS delivered after $120 \mathrm{~s}$ of stimulation (Fig. $5 E$ ), but slower than the rate of decay from rest. This is congruent with the main decrease in probability of release occurring during the first 50 events. Finally, the bottom plots illustrate how the time course of IPSC amplitudes normalized to the first HFS event is similar after 50 events or $120 \mathrm{~s}$ of stimulation, although different for BF 10 or $67 \mathrm{~Hz}$ (left and right, respectively, i.e., higher relative facilitation at the beginning of HFS delivered from BF $10 \mathrm{~Hz}$ than from $67 \mathrm{~Hz}$ ). These results suggest that the proportions of fast facilitation and depression recruited by changes in rate differ depending on the $\mathrm{BF}(10$ or $67 \mathrm{~Hz})$, but importantly, this proportion is not altered by S-STD expression. This supports the view that the SSTD mechanism is distinct and behaves independently from the processes underlying faster forms of plasticity.

Together, these results strongly suggest that S-STD is inputspecific, involves a presynaptic mechanism, probably a change in the number of available quanta $(N)$, and its mechanism does not interfere with the balance of faster forms of synaptic plasticity.

\section{A model with an independent slow-depression component explains well the experimental results}

Computer simulations were used to further gain insight into how different synaptic processes could interact to explain the present results. As a starting point, I tested a version of previously published models with two different pools of ready-to-bereleased vesicles (RRPs) (Trommershauser et al., 2003), which successfully explained PC_DCN responses to trains of hundreds of stimuli (Turecek et al., 2016). Briefly, this model requires two different RRPs (RRPA with high probability of release (Pr) and slow recovery time constant $\left(\mathrm{Tau}_{\mathrm{R}}\right)$; and RRPB with low Pr, fast $\mathrm{Tau}_{\mathrm{R}}$, and facilitation) to explain PC_DCNs initial fast phase of depression, the substantial output with continuous activation, and the phase of frequency invariance (Turecek et al., 2016) (for further details, see Materials and Methods; Fig. 6, scheme). This model (hereafter D + F model; Fig. 6) explained well the present PC_DCN responses to the first 100 stimuli of trains with frequencies 10, 29.5, and $67 \mathrm{~Hz}$ (Fig. 6, same data as in Fig. 1), including frequency invariance phases and approximately linear encoding explained by the counteracting fast facilitation and depression of release, confirming previous results (Turecek et al., 2016).

However, the D $+\mathrm{F}$ model could not explain the further decay in IPSC amplitude with protracted stimulation (Fig. 7A), even when facilitation was omitted. The mechanism(s) of slow depression is debated, but it has been recently proposed that, under intense or sustained synaptic activity, the most sensitive bottleneck for the availability of vesicles for release is the number of release sites that after undergoing exocytosis are cleared and prepared to receive new vesicles or quanta (Neher, 2010). Considering the latter idea, the frequency dependence of S-STD, the evidence that S-STD depends on decrease in available quanta, and that is independent from fast forms of plasticity, I implemented a slow-depression component simulated by an activitydependent decrease in active release sites of Pool B (R_SitesB), recovering with fixed time constant (Fig. $7 B$ ) (see Materials and Methods). The properties of the release sites remaining active are unchanged by this process. Inclusion of this component extended the $\mathrm{D}+\mathrm{F}$ model. The extended model (hereafter, SD_RS model) was able to explain the changes in IPSC amplitude observed during 120-s-long stimulation trains (Fig. 7B). Importantly, this model explained as well the results from a different set of experiments using a switch in the protracted stimulation frequency within the same trial (i.e., from 10 to $67 \mathrm{~Hz}$ ) (Fig. 7C, details in Fig. 8). The experimental results in Figure $7 C$ confirmed those obtained using cell-attached recordings (Fig. 


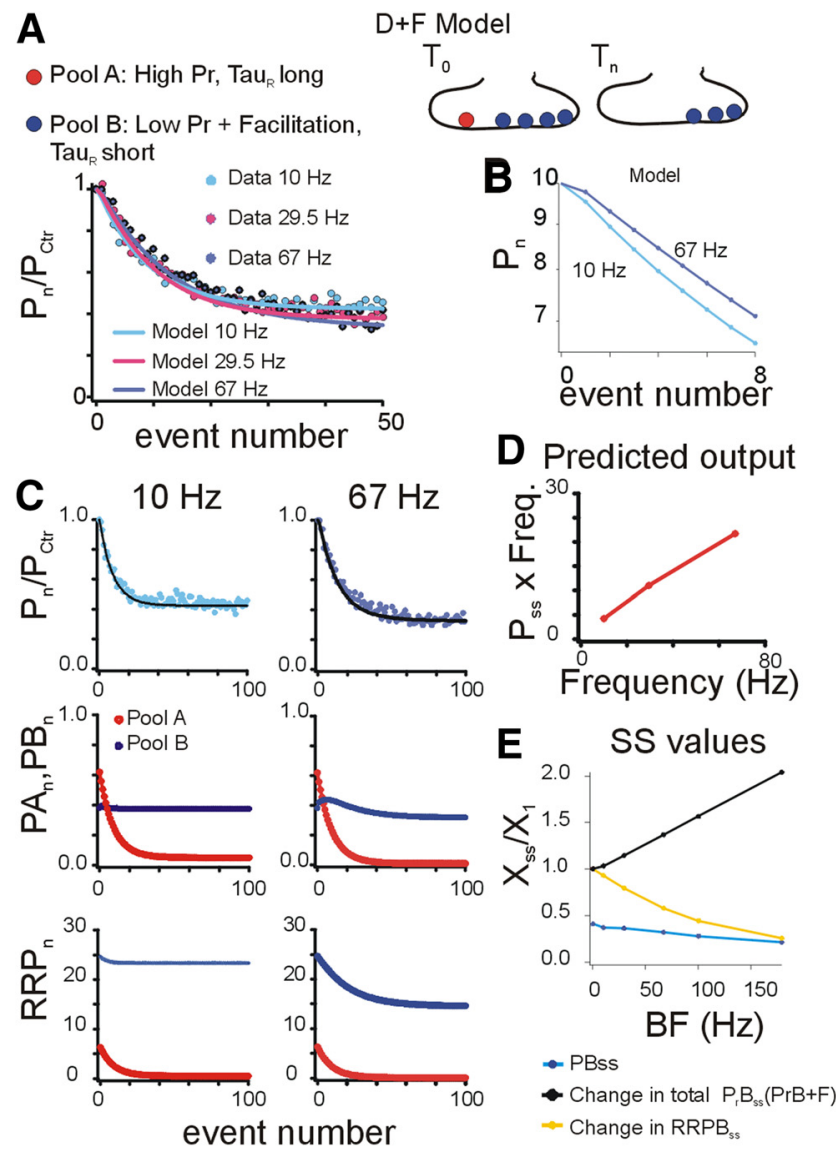

Figure 6. Experimental and simulated responses of a two-pool and facilitation model of PC_DCNs to different BFs. $A$, Top, D + F model scheme. Synapses contain two different pools of vesicles $A$ and $B$, which differ in release probability $(\mathrm{Pr})$, time constant of recovery $\left(\operatorname{Tau}_{R}\right)$, and presence of facilitation. Bottom, The predicted output of a two-pool and facilitation model ( $D+F$ model) explains the experimental responses to the first 100 events of stimulation trains at $10,29.5$, and $67 \mathrm{~Hz}$ (same data as in Fig. 1). $\boldsymbol{B}$, Detail of the model responses to the first 8 events of the trains at 10 and $67 \mathrm{~Hz}$ using a semilogarithmic plot to illustrate slower decaying rates with higher stimulating frequencies. $\boldsymbol{C}$, Left and right plots (for 10 and $67 \mathrm{~Hz}$ stimulation rates, respectively) represent, from top to bottom, the data (circles) and model output (continuous lines), the predicted IPSC amplitudes for Pools $A$ and B (PA, PB, red and blue), and the size of the ready to be released $A$ and $B$ pools (RRPA, RRPB, red and blue, respectively). $\boldsymbol{D}$, The relationship of the predicted synaptic output at steady state (estimated by the product of the predicted IPSC amplitude and the stimulation frequency) versus the stimulation frequency is almost linear. $\boldsymbol{E}$, Summary of results predicted by the $D+F$ model at steady state as a function of the stimulation frequency: the predicted output of the Pool B (PB, blue circles), the total probability of release of the Pool B normalized to Event 1 $\left(P_{r} B+\right.$ facilitation, black circles), and the change in RRPB (yellow circles), normalized to Event 1. $\operatorname{RRPA}_{0}=7, \mathrm{RRPB}_{0}=25, \operatorname{Pr} A=0.098, \operatorname{Pr} B=0.017$, $\operatorname{Tau}_{R} A=12, \operatorname{Tau}_{R} B=0.5$, $\mathrm{f} 1=0.0005$, Tauf1 $=0.007, \mathrm{f} 2=0.001$, Tauf2 $=0.1$.

$3 E, F)$, further demonstrating that S-STD adjusts slowly to new levels, requiring several tens of seconds to reach a new steady state. In the model, the slow changes in the number of "active release sites" after the switch correlated with the slow time course of depression (R_Sites B, Fig. 8D, inset). Note the similarity in the time course of the amplitudes of the experimental and predicted responses: first, a relative facilitation of IPSCs amplitude follows the switch, recovers, and afterward slowly readapts up to a new (lower) steady-state level.

The comparison of steady-state values of the normalized RRPB size (NRRPB, diamonds) and the output of Pool B (PB, circles) predicted by the SD_RS (blue) and the D + F (yellow) models, which differ only by the presence or absence of the slowdepression component, highlights the effect of the latter (Fig. 7D, left). The plot also illustrates the frequency-dependent change in the slow-depression component represented by the fractional size of active release sites of Pool $\mathrm{B}\left(\mathrm{R} \_\right.$Sites $\mathrm{B}_{\mathrm{ss}}$ normalized to the value at $\mathrm{P}_{0}, \mathrm{NR}$ _SitesB, red triangles). The scaling effect of S-STD (Fig. 4A) suggested a multiplicative relationship of the slowdepression component. The here implemented inactivation of sites does not interfere with the properties of the remaining active ones, predicting a pure scaling effect. Indeed, the product of the NRRPB or the PB values of the $\mathrm{D}+\mathrm{F}$ model, times the slow-depression component (NR_Sites $B_{s s}$ ), returned the RRPB and $\mathrm{PB}$ values of the $\mathrm{SD} \_\mathrm{RS}$ model, respectively (Fig. $7 D$, right, respective products shown in light blue), graphically illustrating the multiplicative operation of the slow-depression component for steady-state conditions. Noteworthy, due to the minimal contribution of Pool A to the total output at steady state, similar results emerged from the analysis of the total output $(\mathrm{PA}+\mathrm{PB})$ (Fig. 8E). Therefore, the extended model explained both the changes in amplitude and the time course of these changes during protracted stimulation and after a switch in $\mathrm{BF}$.

Next, I checked whether this model accounted as well for the $B F-d e p e n d e n t$ changes in gain for the responses to test trains (e.g., Fig. 4A). The predictions of both models to steps of changes in activation rate (duration: $0.5 \mathrm{~s}$ ) induced after sustained activation, as applied experimentally to PC_DCN synapses (Fig. 7E1), were investigated. As expected, the integral of the predicted output over the step duration (proxy for the charge transfer amplitude) by the D + F model increased almost linearly as a function of the step frequency up to $200 \mathrm{~Hz}$; however, in contrast to the experimental results, the slopes or gains for different BFs were almost coincident (Fig. 7E2, left vs Fig. 4A). Instead, the predictions by the SD_RS model (Fig. 7E2, right) showed similar linear increases in output but clearly distinct slopes for different BFs, indicating that the model captures the changes in gain mediated by the different BFs. Moreover, dividing the values obtained with the SD_RS model by the normalized number of release sites present at steady state before the step returned the values of the $\mathrm{D}+\mathrm{F}$ model (Fig. 7E3), indicating that the multiplicative effect of this slow-depression component of Pool B was sufficient to explain the effect. Furthermore, the number of active release sites changed slowly (Fig. $8 D$ ), explaining that the number of sites available just before the step determines the gain during the post-ceding subsecond-to-few seconds periods of the steps in frequency. The fast STP components (given by the total facilitation and RRP depletion) led to opposing effects during the step (Fig. 8), contributing to the linear encoding during this time window.

Summarizing, a model of synaptic transmission, including a slow-depression component with a multiplicative relationship to the simulated fast plasticity components, explains well the experimental data. Moreover, these results demonstrate that an activity-dependent change in release sites is a plausible molecular mechanism for slow depression at PC_DCN synapses and consequent gain control.

\section{Discussion}

This study examined how cerebellar PCs, which are continuously active, can faithfully transfer behavior-driven signals embedded in the incessant background activity and this, without driving the postsynaptic DCNs out of range. By using protracted PC_DCN activation, I identified a previously unrecognized frequencydependent S-STD, which requires tens of seconds or thousands of events to reach or readjust to new steady-state levels, adapting PC_DCNs output to the background activity. Yet, PC stimulation 


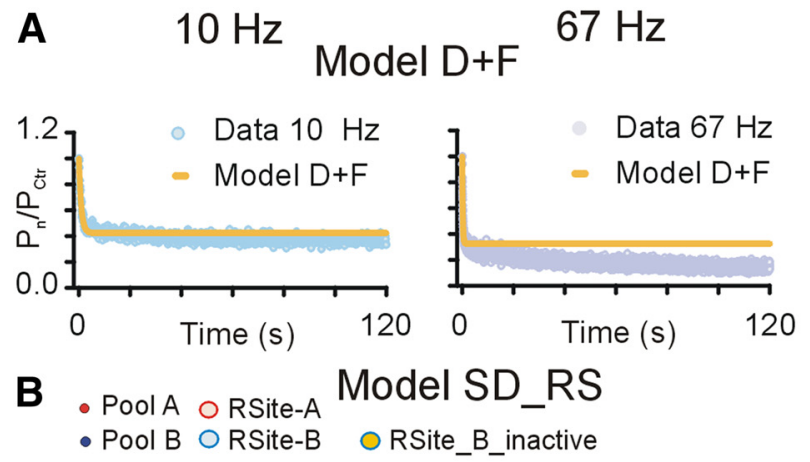

D $\quad$ Model responses at SS
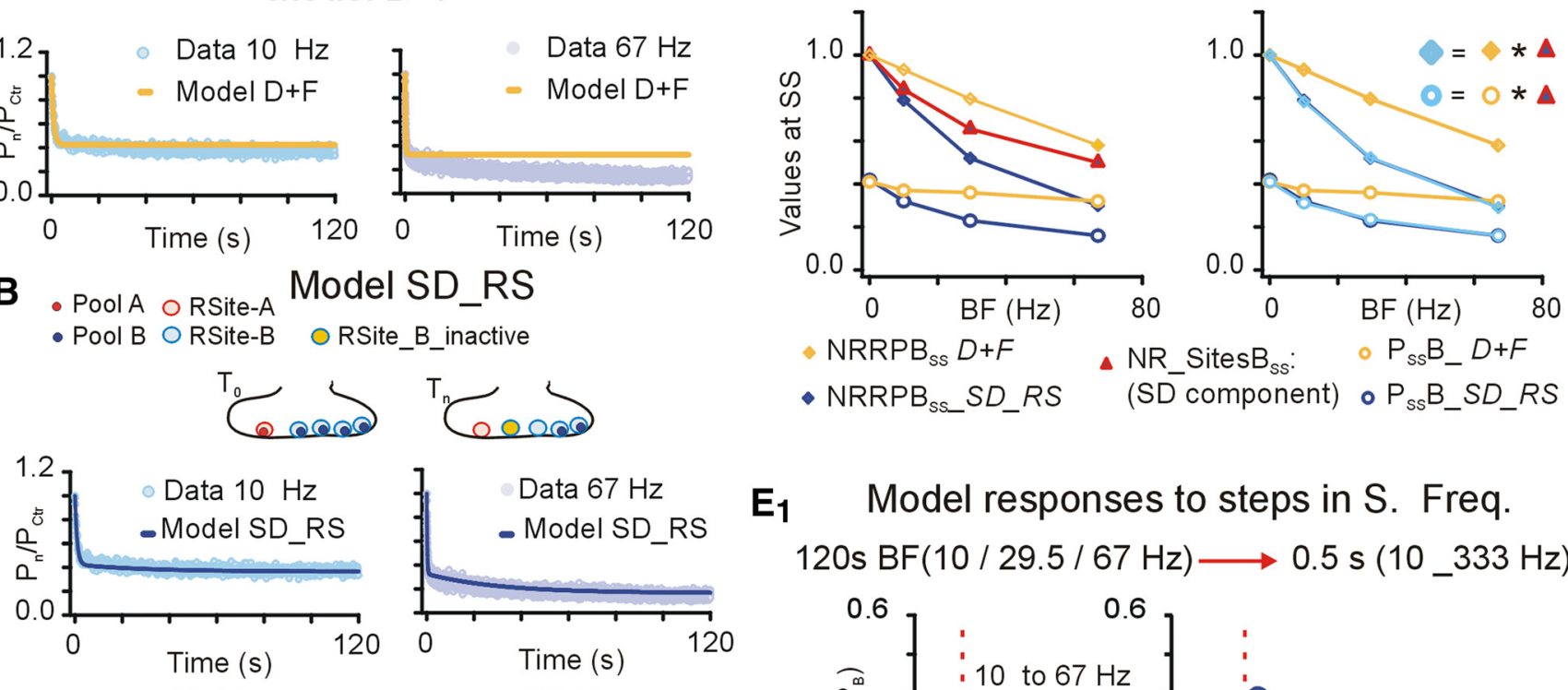

$$
\begin{array}{lll}
-N_{R R P B} D+F & \Delta R \_S_{s s} D+B_{s s}: & \circ P_{s s} B_{-} D+F \\
-N_{R R P B} B_{s s} S D_{-} R S & \text { (SD component) } & \circ P_{s s} B_{-} S D_{-} R S
\end{array}
$$

\section{$\mathbf{E}_{1} \quad$ Model responses to steps in S. Freq. $120 \mathrm{~s} B F(10 / 29.5 / 67 \mathrm{~Hz}) \longrightarrow 0.5 \mathrm{~s}\left(10 \_333 \mathrm{~Hz}\right)$}

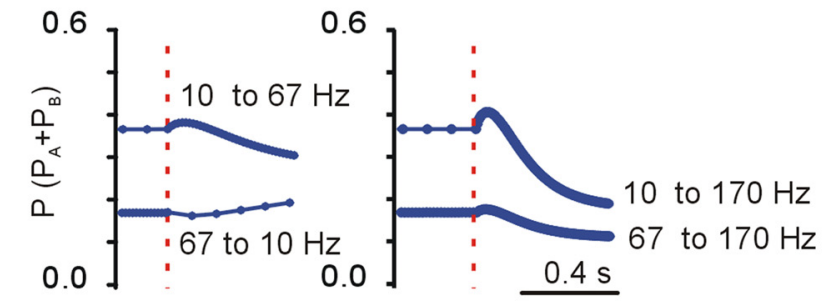

E2
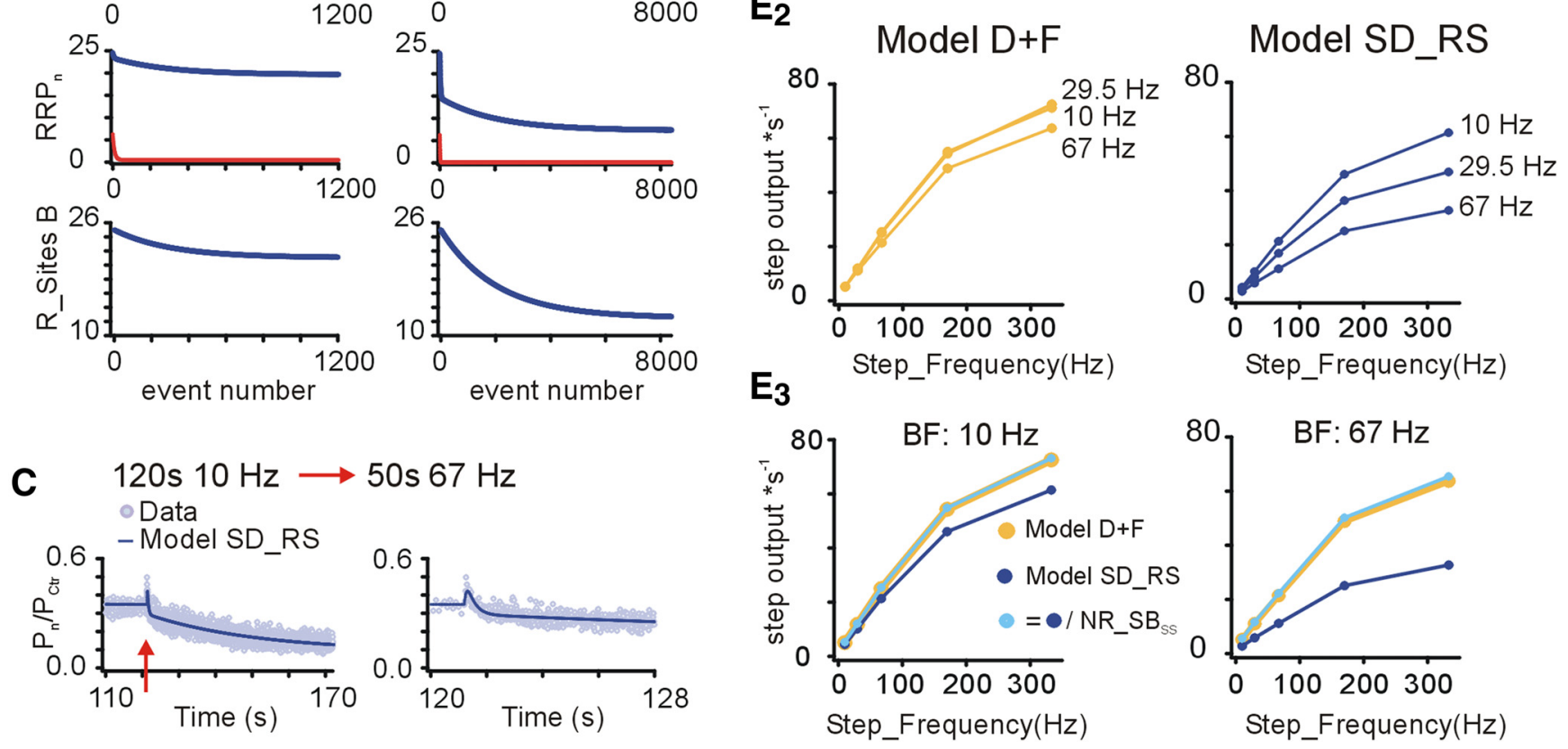

Figure 7. A two-pool and facilitation model extended by a slow-depression component (SD) explains experimental S-STD and scaled linear encoding of responses to $0.5 \mathrm{~s}$ steps of changes in rate. $A$, Predictions from a two-pool and facilitation model ( $D+F$ model) (yellow line, same fitting parameters as in Fig. 6) fail to explain S-STD (experimental data: blue and violet circles represent 10 and $67 \mathrm{~Hz}$, respectively; same data as in Fig. 1). $\boldsymbol{B}$, Instead, both, early and late experimental responses were explained by a model featuring a slow-depression (SD) component simulating an activity-dependent decrease in number of active release sites of Pool B (ready to be refilled after use and filled), schematized on top. Bottom, Data and model output (SD-RS model: dark blue line; see Materials and Methods, data as in $A$ ). In lower rows from top to bottom (Pool A in red and Pool B in blue): the predicted IPSC amplitudes of Pools A and B (PA, PB), the size of (filled) ready-to-be-released A and B pools (RRPA, RRPB), and the number of active release sites of Pool B (R_SitesB). C, Experimental (light blue) and SD-RS model predictions (dark blue line) to a sustained change in stimulation frequency (120 s at $10 \mathrm{~Hz}$ followed by $50 \mathrm{~s}$ at $67 \mathrm{~Hz}$, indicated by the top scheme). Right, Detail in expanded time scale, around the switch time. Data from a different set of neurons than $\boldsymbol{A}$ and $\boldsymbol{B}(n=6)$; the model estimated parameters are in Figure 8. $\boldsymbol{D}$, Left, Summary of predicted steady-state normalized values of RRPB (NRRPB, filled squares) and PB (open circles) by the D + F (yellow) and SD_RS (dark blue) models and of the slow-depression component (red triangles, R_SitesB of the RS-SD model, normalized to the value at $\mathrm{PO}$ ) as a function of the BF from experiments as in $\boldsymbol{A}$ and $\boldsymbol{B}$. Right, a multiplicative effect of the SD component explains the differences between the models. Light blue curves indicate the product of the $D+F$ curves (yellow) times the corresponding fractional R_SitesB (shown on the left plot in red). Dark blue traces (SD_RS model predictions) are occluded by the light blue curves. $\boldsymbol{E}_{1}$, Model predictions to steps of change in stimulating frequency (proxy for the test trains in Fig. 4). Top, Stimulation paradigm. Bottom, Examples of SD_RS model responses. Red dashed line indicates beginning of the step. $\boldsymbol{E}_{1}$, Plots of the integral of predicted IPSCs (proxy for charge transfer) as a function of the step frequency, for different BFs (labeled on the right). The D + F and SD_RS model outputs correspond to the left and right plots, respectively. $E_{3}$, Multiplicative effect of SD: predicted input/output functions by the D + F (yellow) 
paradigms mimicking behavior-related signals embedded in the background activity revealed a subsecond to second-long time window during which the evoked charge transfer or changes in DCN firing rate were proportional to the PC rates, with gain dependent on preceding background activity. Thus, S-STD supports a novel slowgain control mechanism enabling faithful linear encoding of $\mathrm{PC}$ rates in the timescale of common movements (e.g., reaches, steps) and adaptation to background activity at longer timescales. Experimental evidence and simulations suggested the scaled linear encoding emerged from the combined S-STD slow dynamics and frequency-invariant gain at faster time scales. Mechanistically, a presynaptic mechanism based on decreased ready-to-release vesicles, distinct from faster plasticity forms, plausibly, a reduction in the number of active release sites, could explain S-STD.

The use of protracted PC_DCN activation has been key in the identification of S-STD, which was a robust finding, revealed using regular and irregular activation patterns, in preparations from young or adult animals, and under conditions approximating those found in situ (i.e., temperature, calcium concentration). Furthermore, the consequences of S-STD were readily assessable measuring synaptic charge transfer or DCN changes in firing rate, confirming its functional significance. Since protracted synaptic activation tests are rarely used, the prevalence of slow depression at other synapses is uncertain. Evidence for S-STD was first recognized at the neuromuscular junction (Del Castillo and Katz, 1954; Elmqvist and Quastel, 1965) and subsequently in hippocampal cell cultures, hippocampal slices, and the auditory pathway (Liu and Tsien, 1995; Stevens and Wesseling, 1999; Garcia-Perez et al., 2008; Hennig et al., 2008; Krächan et al., 2017). However, functional S-STD expression requires sustained activation, a condition clearly met at PC_DCNs (Sato et al., 1992; Norris et al., 2004; Schonewille et al., 2006; Cao et al., 2012; Hong et al., 2016), but apparently not at hippocampal synapses (Klyachko and Stevens, 2006). Still, as sustained activity is central to several brain processes, the relevance of the present findings might bear relevance to other brain functions.

Several lines of evidence argue that S-STD is distinct from all forms of depression previously described at PC_DCNs. First, SSTD is more than one order of magnitude slower than previously reported forms of depression at these synapses (Telgkamp and Raman, 2002; Pedroarena and Schwarz, 2003; Najac and Raman, 2015; Turecek et al., 2016), requiring tens of seconds to minutes, instead of milliseconds to seconds, to reach steady state or to adjust to new levels after changes in rate (Figs. 1, 3, 5, and 7).

\section{$\leftarrow$}

and SD-RS (dark blue) models as in $E_{2}$, for 10 and $67 \mathrm{~Hz}$ BF (left and right plots, respectively). Light blue traces were obtained by dividing the output of the SD-RS model (dark blue) by the corresponding fraction of $R$ _ Sites $B$ available before the frequency step. (SD-RS: $\mathrm{RRPA}_{0}=7, \mathrm{RRPB}_{0}=25, \operatorname{PrA}=0.098, \operatorname{PrB}=0.017, \mathrm{Tau}_{\mathrm{RA}}=12, \mathrm{Tau}_{\mathrm{RB}}=0,5, \mathrm{f} 1=0.0005$, Tauf1 $=0.007, \mathrm{f2}=0.001$, Tauf2 $=0.1, \mathrm{ARSB}=0.47, \mathrm{~F} \_\mathrm{RS}=29$, TauRSr $=30$, apply to all panels except Fig. 6 C, see details in main text).
Second, the relative proportion of fast facilitation/depression evoked by test trains is similar before and after S-STD induction (Fig. 5F). Moreover, S-STD enables linear encoding of PC rates, presumed to result from balanced fast facilitation and depression (Turecek et al., 2016) (Fig. 4). Finally, simulations using an SSTD component multiplicatively related to fast facilitation/ depression ones explain the experimental responses to diverse stimulation paradigms, further supporting the idea that S-STD is different from faster forms of PC_DCN plasticity.

The mechanism of S-STD is debated, with proposed mechanisms including depletion of a reserve pool (Elmqvist and Quastel, 1965; Richards et al., 2003), "fatigue" in the replenishment of the readily releasable pool of vesicles (Gabriel et al., 2011), and decreased release probability (Hennig et al., 2008). The latter mechanism seems unlikely for PC_DCNs given the analysis of depression rates and $\mathrm{CV}^{-2}$ (Fig. 5) (but see Faber and Korn, 1991). The same results speak against a decreased quantal size, for example, by incomplete vesicle refilling (Bennett et al., 1976) or postsynaptic change in the chloride gradient. Furthermore, $\mathrm{GABA}_{\mathrm{B}}$-dependent slow gain control (Magnusson et al., 2008) is unlikely the cause for PC_DCN S-STD as their respective time courses differ, and S-STD modulation persists under $\mathrm{GABA}_{\mathrm{B}}$ antagonists, in line with previous results (Pedroarena and Schwarz, 2003). Recently, the availability of "receptive" release sites has been proposed to be the principal bottleneck for the maintenance of intense sustained synaptic transmission (Neher, 2010), suggested by observations of rapid reduced synaptic release under unbalanced exocytosis and endocytosis, faster than expected from a failure in vesicle recycling (Kawasaki et al., 2000; Hosoi et al., 2009). The exact mechanism limiting release site availability is not clear (for review, see Byczkowicz et al., 2018) but could explain S-STD. Indeed, changes in release sites was proposed earlier to explain a slow phase of recovery from intense activity (Stevens and Wesseling, 1999). However, S-STD must not be universally obligatory (Mayer et al., 2014), as other synaptic processes or properties (Kalkstein and Magleby, 2004; Kandaswamy et al., 2010; Byczkowicz et al., 2018; Xue et al., 2018) could mitigate the release site limitation. In the present study, I implemented "release site availability" as a component of a previously published synaptic transmission model explaining PC_DCN responses 
on shorter time scales (Turecek et al., 2016). The success of this extended model in predicting experimental S-STD and scaled linear transmission strongly favored "release site availability" as PC_DCN S-STD mechanism. The change in "release site availability" sites does not (necessarily) influence the properties of the remaining sites and thus is compatible with present evidence that S-STD expression does not interfere with faster plasticity processes. Here, the rate at which single vesicle-depleted release sites refill was kept constant, although activity-dependent refill (Pan and Zucker, 2009) could explain supralinear responses evoked by brief high frequency PC stimulation (Fig. $4 F)$. The total refilling rate varied here only due to changes in the total number of "accepting release sites." Overall, this study supports a decrease in "accepting release sites" as a plausible mechanism for S-STD and (together with fast frequency-invariant synaptic transmission) the derived slow-gain control mechanism described here. Nonetheless, the molecular identity behind the S-STD phenomenon could correspond to an equivalent process, and recent advances warrant further studies (Doussau et al., 2017; Milovanovic et al., 2018; Patzke et al., 2019; Vaden et al., 2019).

What functional consequences arise from PC_DCN-S-STD? First, the mere presence of S-STD and resulting shallow relationship between the background-stimulation frequency $(\mathrm{BF})$ and steady-state output (Fig. $5 C$, inset) suggests that S-STD deemphasizes the effect of PC background activity in behaving animals. Thus, S-STD could partly explain previous findings of little sensitivity of DCN firing responses to sustained changes PC_BF (Belmeguenai et al., 2010). The decreased sensitivity to steady PC rates could be useful for maintaining the inhibitory PC output within the working firing range of DCNs, avoiding transmission failure (Pedroarena, 2010).

Second, S-STD offers a period of stable gain supporting scaled linear encoding of PC rates in the PC_DCNs synaptic output over behavior-relevant time windows and in the firing rates of large DCNs. Because DCN rate is less affected by synchronized than desynchronized PC inputs (Sarnaik and Raman, 2018), the present results suggest that rate code could be robust under more physiological desynchronized conditions. The findings are in line with in vivo OG manipulation studies showing inverse variations in PC and DCN rates (Heiney et al., 2014; Ozcan et al., 2020). Moreover, the fact that S-STD guarantees similar outputs from PCs with widely varying background activity favors linear encoding of changes in rate at the PC population level (Fig. 4) (Abbott et al., 1997). Noteworthy, although PC_DCN linear encoding was proposed as advantageous for cerebellar computations, the period of stable gain provided by S-STD could also support other previously proposed PC_DCN encoding strategies (Shidara et al., 1993; Walter and Khodakhah, 2009; De Zeeuw et al., 2011; Heck et al., 2013): for example, transferring the timing of synchronized (complex or simple) spikes, or firing pauses (Welsh et al., 1995; Gauck and Jaeger, 2000; Shin et al., 2007; Hoebeek et al., 2010; Person and Raman, 2012; Hong et al., 2016; Tang et al., 2019; Ozcan et al., 2020), or inducing binary or graded signal inversion (Llinás and Mühlethaler, 1988; Pedroarena, 2010; Boehme et al., 2011; Ten Brinke et al., 2017). In this line, findings of preferential transfer of brief PC signals (Fig. 4F) suggest that PC_DCNs could discriminate signal length or timing, promoting the expression of temporal codes.

Finally, and intriguingly, the S-STD mediated changes in gain without changes in the linear transfer function for stimuli mimicking behavior-driven/learned PC signals is reminiscent, at synaptic level, of the gain modulation of neuronal population responses (Salinas and Thier, 2000), a computation proposed to account for nonlinear combination of signals (Salinas, 2004). PC sustained firing and PC behavior-related signals converge at single synapses, but each may represent different information and, consequently, change independently. Long-term or transient modifications of PC background activity could modulate PC_DCN gain for stereotyped signals, such as complex spikes (see Fig. $4 A, B$ ), providing an alternative and flexible mechanism to adapt PC output at the last cerebellar stage (Titley et al., 2017), revealing a novel role for PC background activity in controlling cerebellar output.

\section{References}

Abbasi S, Hudson AE, Maran SK, Cao Y, Abbasi A, Heck DH, Jaeger D (2017) Robust transmission of rate coding in the inhibitory Purkinje cell to cerebellar nuclei pathway in awake mice. PLoS Comput Biol 13: e1005578.

Abbott LF, Varela JA, Sen K, Nelson SB (1997) Synaptic depression and cortical gain control. Science 275:220-224.

Arenz A, Silver RA, Schaefer AT, Margrie TW (2008) The contribution of single synapses to sensory representation in vivo. Science 321:977-980.

Bekkers JM, Stevens CF (1990) Presynaptic mechanism for long-term potentiation in the hippocampus. Nature 346:724-729.

Belmeguenai A, Hosy E, Bengtsson F, Pedroarena CM, Piochon C, Teuling E, He Q, Ohtsuki G, De Jeu MT, Elgersma Y, De Zeeuw CI, Jörntell H, Hansel C (2010) Intrinsic plasticity complements long-term potentiation in parallel fiber input gain control in cerebellar Purkinje cells. J Neurosci 30:13630-13643.

Bennett MV, Model PG, Highstein SM (1976) Stimulation-induced depletion of vesicles, fatigue of transmission and recovery processes at a vertebrate central synapse. Cold Spring Harb Symp Quant Biol 40:25-35.

Boehme R, Uebele VN, Renger JJ, Pedroarena C (2011) Rebound excitation triggered by synaptic inhibition in cerebellar nuclear neurons is suppressed by selective T-type calcium channel block. J Neurophysiol 106:2653-2661.

Borst JG (2010) The low synaptic release probability in vivo. Trends Neurosci 33:259-266.

Bryant JL, Roy S, Heck DH (2009) A technique for stereotaxic recordings of neuronal activity in awake, head-restrained mice. J Neurosci Methods 178:75-79.

Buchs NJ, Senn W (2002) Spike-based synaptic plasticity and the emergence of direction selective simple cells: simulation results. J Comput Neurosci 13:167-186.

Byczkowicz N, Ritzau-Jost A, Delvendahl I, Hallermann S (2018) How to maintain active zone integrity during high frequency transmission. Neurosci Res 127:61-69.

Cao Y, Maran SK, Dhamala M, Jaeger D, Heck DH (2012) Behavior-related pauses in simple-spike activity of mouse Purkinje cells are linked to spike rate modulation. J Neurosci 32:8678-8685.

Catz N, Dicke PW, Thier P (2008) Cerebellar-dependent motor learning is based on pruning a Purkinje cell population response. Proc Natl Acad Sci USA 105:7309-7314.

Chang S, Trimbuch T, Rosenmund C (2018) Synaptotagmin-1 drives synchronous $\mathrm{Ca}(2+)$-triggered fusion by $\mathrm{C} 2 \mathrm{~B}$-domain-mediated synapticvesicle-membrane attachment. Nat Neurosci 21:33-40.

Chaumont J, Guyon N, Valera AM, Dugué GP, Popa D, Marcaggi P, Gautheron V, Reibel-Foisset S, Dieudonné S, Stephan A, Barrot M, Cassel JC, Dupont JL, Doussau F, Poulain B, Selimi F, Léna C, Isope P (2013) Clusters of cerebellar Purkinje cells control their afferent climbing fiber discharge. Proc Natl Acad Sci USA 110:16223-16228.

Chen C, Satterfield R, Young SM Jr, Jonas P (2017) Triple function of synaptotagmin 7 ensures efficiency of high frequency transmission at central GABAergic synapses. Cell Rep 21:2082-2089.

Chen S, Augustine GJ, Chadderton P (2016) The cerebellum linearly encodes whisker position during voluntary movement. eLife 5:e10509.

De Zeeuw CI, Wylie DR, Stahl JS, Simpson JI (1995) Phase relations of Purkinje cells in the rabbit flocculus during compensatory eye movements. J Neurophysiol 74:2051-2064. 
De Zeeuw CI, Hoebeek FE, Bosman LW, Schonewille M, Witter L, Koekkoek SK (2011) Spatiotemporal firing patterns in the cerebellum. Nat Rev Neurosci 12:327-344.

Del Castillo J, Katz B (1954) Statistical factors involved in neuromuscular facilitation and depression. J Physiol 124:574-585.

Doussau F, Schmidt H, Dorgans K, Valera AM, Poulain B, Isope P (2017) Frequency-dependent mobilization of heterogeneous pools of synaptic vesicles shapes presynaptic plasticity. Elife 6:e28935.

Dugué GP, Tihy M, Gourévitch B, Léna C (2017) Cerebellar re-encoding of self-generated head movements. eLife 6:e26179.

Elmqvist D, Quastel DM (1965) A quantitative study of end-plate potentials in isolated human muscle. J Physiol 178:505-529.

Faber DS, Korn H (1991) Applicability of the coefficient of variation method for analyzing synaptic plasticity. Biophys J 60:1288-1294.

Fekete A, Nakamura Y, Yang YM, Herlitze S, Mark MD, DiGregorio DA, Wang LY (2019) Underpinning heterogeneity in synaptic transmission by presynaptic ensembles of distinct morphological modules. Nat Commun 10:826.

Gabriel T, García-Pérez E, Mahfooz K, Goñi J, Martínez-Turrillas R, PérezOtaño I, Lo DC, Wesseling JF (2011) A new kinetic framework for synaptic vesicle trafficking tested in synapsin knock-outs. J Neurosci 31:1156311577.

Gao Z, van Beugen BJ, De Zeeuw CI (2012) Distributed synergistic plasticity and cerebellar learning. Nat Rev Neurosci 13:619-635.

Garcia-Perez E, Lo DC, Wesseling JF (2008) Kinetic isolation of a slowly recovering component of short-term depression during exhaustive use at excitatory hippocampal synapses. J Neurophysiol 100:781-795.

Gauck V, Jaeger D (2000) The control of rate and timing of spikes in the deep cerebellar nuclei by inhibition. J Neurosci 20:3006-3016.

Grasselli G, Boele HJ, Titley HK, Bradford N, van Beers L, Jay L, Beekhof GC, Busch SE, de Zeeuw CI, Schonewille M, Hansel C (2020) SK2 channels in cerebellar Purkinje cells contribute to excitability modulation in motor-learning-specific memory traces. PLoS Biol 18:e3000596.

Halverson HE, Khilkevich A, Mauk MD (2015) Relating cerebellar Purkinje cell activity to the timing and amplitude of conditioned eyelid responses. J Neurosci 35:7813-7832.

Heck DH, De Zeeuw CI, Jaeger D, Khodakhah K, Person AL (2013) The neuronal code(s) of the cerebellum. J Neurosci 33:17603-17609.

Heiney SA, Kim J, Augustine GJ, Medina JF (2014) Precise control of movement kinematics by optogenetic inhibition of Purkinje cell activity. J Neurosci 34:2321-2330.

Hennig MH, Postlethwaite M, Forsythe ID, Graham BP (2008) Interactions between multiple sources of short-term plasticity during evoked and spontaneous activity at the rat calyx of Held. J Physiol 586:3129-3146.

Herzfeld DJ, Kojima Y, Soetedjo R, Shadmehr R (2015) Encoding of action by the Purkinje cells of the cerebellum. Nature 526:439-442.

Hoebeek FE, Witter L, Ruigrok TJ, De Zeeuw CI (2010) Differential olivocerebellar cortical control of rebound activity in the cerebellar nuclei. Proc Natl Acad Sci USA 107:8410-8415.

Hong S, Negrello M, Junker M, Smilgin A, Thier P, De Schutter E (2016) Multiplexed coding by cerebellar Purkinje neurons. eLife 5:e13810.

Hosoi N, Holt M, Sakaba T (2009) Calcium dependence of exo- and endocytotic coupling at a glutamatergic synapse. Neuron 63:216-229.

Jelitai M, Puggioni P, Ishikawa T, Rinaldi A, Duguid I (2016) Dendritic excitation-inhibition balance shapes cerebellar output during motor behaviour. Nat Commun 7:13722.

Kalkstein JM, Magleby KL (2004) Augmentation increases vesicular release probability in the presence of masking depression at the frog neuromuscular junction. J Neurosci 24:11391-11403.

Kandaswamy U, Deng PY, Stevens CF, Klyachko VA (2010) The role of presynaptic dynamics in processing of natural spike trains in hippocampal synapses. J Neurosci 30:15904-15914.

Kawasaki F, Hazen M, Ordway RW (2000) Fast synaptic fatigue in Shibire mutants reveals a rapid requirement for dynamin in synaptic vesicle membrane trafficking. Nat Neurosci 3:859-860.

Klyachko VA, Stevens CF (2006) Excitatory and feed-forward inhibitory hippocampal synapses work synergistically as an adaptive filter of natural spike trains. PLoS Biol 4:e207.

Krächan EG, Fischer AU, Franke J, Friauf E (2017) Synaptic reliability and temporal precision are achieved via high quantal content and effective replenishment: auditory brainstem versus hippocampus. J Physiol 595:839-864.
Laurens J, Angelaki DE (2017) A unified internal model theory to resolve the paradox of active versus passive self-motion sensation. Elife 6:e28074.

Linnemann C, Sultan F, Pedroarena CM, Schwarz C, Thier P (2004) Lurcher mice exhibit potentiation of GABA(A)-receptor-mediated conductance in cerebellar nuclei neurons in close temporal relationship to Purkinje cell death. J Neurophysiol 91:1102-1107.

Liu G, Tsien RW (1995) Properties of synaptic transmission at single hippocampal synaptic boutons. Nature 375:404-408.

Llinás R, Mühlethaler M (1988) Electrophysiology of guinea-pig cerebellar nuclear cells in the in vitro brain stem-cerebellar preparation. J Physiol 404:241-258.

MacLeod KM, Horiuchi TK, Carr CE (2007) A role for short-term synaptic facilitation and depression in the processing of intensity information in the auditory brain stem. J Neurophysiol 97:2863-2874.

Magleby KL (1973) The effect of tetanic and post-tetanic potentiation on facilitation of transmitter release at the frog neuromuscular junction. J Physiol 234:353-371.

Magnusson AK, Park TJ, Pecka M, Grothe B, Koch U (2008) Retrograde GABA signaling adjusts sound localization by balancing excitation and inhibition in the brainstem. Neuron 59:125-137.

Malinow R, Tsien RW (1990) Presynaptic enhancement shown by whole-cell recordings of long- term potentiation in hippocampal slices. Nature 346:177-180.

Mayer F, Albrecht O, Dondzillo A, Klug A (2014) Glycinergic inhibition to the medial nucleus of the trapezoid body shows prominent facilitation and can sustain high levels of ongoing activity. J Neurophysiol 112:29012915.

Miki T, Malagon G, Pulido C, Llano I, Neher E, Marty A (2016) Actin- and myosin-dependent vesicle loading of presynaptic docking sites prior to exocytosis. Neuron 91:808-823.

Milovanovic D, Wu Y, Bian X, De Camilli P (2018) A liquid phase of synapsin and lipid vesicles. Science 361:604-607

Najac M, Raman IM (2015) Integration of Purkinje cell inhibition by cerebellar nucleo-olivary neurons. J Neurosci 35:544-549.

Neher E (2010) What is rate-limiting during sustained synaptic activity: vesicle supply or the availability of release sites. Front Synaptic Neurosci 2:144.

Neher E, Sakaba T (2008) Multiple roles of calcium ions in the regulation of neurotransmitter release. Neuron 59:861-872.

Norris SA, Greger B, Hathaway EN, Thach WT (2004) Purkinje cell spike firing in the posterolateral cerebellum: correlation with visual stimulus, oculomotor response, and error feedback. J Neurophysiol 92:1867-1879.

Ozcan OO, Wang X, Binda F, Dorgans K, De Zeeuw CI, Gao Z, Aertsen A, Kumar A, Isope P (2020) Differential coding strategies in glutamatergic and GABAergic neurons in the medial cerebellar nucleus. J Neurosci 40:159-170.

Ozden I, Dombeck DA, Hoogland TM, Tank DW, Wang SS (2012) Widespread state-dependent shifts in cerebellar activity in locomoting mice. PLoS One 7:e42650.

Pan B, Zucker RS (2009) A general model of synaptic transmission and short-term plasticity. Neuron 62:539-554.

Patzke C, Brockmann MM, Dai J, Gan KJ, Grauel MK, Fenske P, Liu Y, Acuna C, Rosenmund C, Sudhof TC (2019) Neuromodulator signaling bidirectionally controls vesicle numbers in human synapses. Cell 179:498-513.e22.

Payne HL, French RL, Guo CC, Nguyen-Vu TB, Manninen T, Raymond JL (2019) Cerebellar Purkinje cells control eye movements with a rapid rate code that is invariant to spike irregularity. Elife 8:e37102.

Pedroarena CM (2010) Mechanisms supporting transfer of inhibitory signals into the spike output of spontaneously firing cerebellar nuclear neurons in vitro. Cerebellum 9:67-76

Pedroarena CM, Schwarz C (2003) Efficacy and short-term plasticity at GABAergic synapses between Purkinje and cerebellar nuclei neurons. J Neurophysiol 89:704-715

Person AL, Raman IM (2012) Synchrony and neural coding in cerebellar circuits. Front Neural Circuits 6:97.

Richards DA, Guatimosim C, Rizzoli SO, Betz WJ (2003) Synaptic vesicle pools at the frog neuromuscular junction. Neuron 39:529-541.

Rinaldo L, Hansel C (2010) Ataxias and cerebellar dysfunction: involvement of synaptic plasticity deficits? Funct Neurol 25:135-139.

Romano V, De Propris L, Bosman LW, Warnaar P, ten Brinke MM, Lindeman S, Ju C, Velauthapillai A, Spanke JK, Middendorp Guerra E, 
Hoogland TM, Negrello M, D'Angelo E, De Zeeuw CI (2018) Potentiation of cerebellar Purkinje cells facilitates whisker reflex adaptation through increased simple spike activity. Elife 7:e38852.

Salinas E (2004) Fast remapping of sensory stimuli onto motor actions on the basis of contextual modulation. J Neurosci 24:1113-1118.

Salinas E, Thier P (2000) Gain modulation: a major computational principle of the central nervous system. Neuron 27:15-21.

Sarnaik R, Raman IM (2018) Control of voluntary and optogenetically perturbed locomotion by spike rate and timing of neurons of the mouse cerebellar nuclei. Elife 7:e29546.

Sato Y, Miura A, Fushiki H, Kawasaki T (1992) Short-term modulation of cerebellar Purkinje cell activity after spontaneous climbing fiber input. J Neurophysiol 68:2051-2062.

Schonewille M, Khosrovani S, Winkelman BH, Hoebeek FE, De Jeu MT, Larsen IM, Van der BJ, Schmolesky MT, Frens MA, De Zeeuw CI (2006) Purkinje cells in awake behaving animals operate at the upstate membrane potential. Nat Neurosci 9:459-461.

Shidara M, Kawano K, Gomi H, Kawato M (1993) Inverse-dynamics model eye movement control by Purkinje cells in the cerebellum. Nature 365:50-52.

Shim HG, Jang DC, Lee J, Chung G, Lee S, Kim YG, Jeon DE, Kim SJ (2017) Long-term depression of intrinsic excitability accompanied by synaptic depression in cerebellar Purkinje cells. J Neurosci 37:5659-5669.

Shin SL, Hoebeek FE, Schonewille M, De Zeeuw CI, Aertsen A, De Schutter E (2007) Regular patterns in cerebellar Purkinje cell simple spike trains. PLoS One 2:e485.

Silver RA (2010) Neuronal arithmetic. Nat Rev Neurosci 11:474-489.

Stevens CF, Wesseling JF (1999) Identification of a novel process limiting the rate of synaptic vesicle cycling at hippocampal synapses. Neuron 24:1017-1028.

Tang T, Blenkinsop TA, Lang EJ (2019) Complex spike synchrony dependent modulation of rat deep cerebellar nuclear activity. Elife 8:e40101.

Telgkamp P, Raman IM (2002) Depression of inhibitory synaptic transmission between Purkinje cells and neurons of the cerebellar nuclei. J Neurosci 22:8447-8457.

Telgkamp P, Padgett DE, Ledoux VA, Woolley CS, Raman IM (2004) Maintenance of high frequency transmission at Purkinje to cerebellar nuclear synapses by spillover from boutons with multiple release sites. Neuron 41:113-126.

Ten Brinke MM, Heiney SA, Wang X, Proietti-Onori M, Boele HJ, Bakermans J, Medina JF, Gao Z, De Zeeuw CI (2017) Dynamic modulation of activity in cerebellar nuclei neurons during pavlovian eyeblink conditioning in mice. Elife 6:e28132.

Thach WT (1968) Discharge of Purkinje and cerebellar nuclear neurons during rapidly alternating arm movements in the monkey. J Neurophysiol 31:785-797.

Titley HK, Brunel N, Hansel C (2017) Toward a neurocentric view of learning. Neuron 95:19-32.
Trommershauser J, Schneggenburger R, Zippelius A, Neher E (2003) Heterogeneous presynaptic release probabilities: functional relevance for short-term plasticity. Biophys J 84:1563-1579.

Tsai PT, Hull C, Chu Y, Greene-Colozzi E, Sadowski AR, Leech JM, Steinberg J, Crawley JN, Regehr WG, Sahin M (2012) Autistic-like behaviour and cerebellar dysfunction in Purkinje cell Tsc1 mutant mice. Nature 488:647-651.

Tsodyks MV, Markram H (1997) The neural code between neocortical pyramidal neurons depends on neurotransmitter release probability. Proc Natl Acad Sci USA 94:719-723.

Turecek J, Jackman SL, Regehr WG (2016) Synaptic specializations support frequency-independent Purkinje cell output from the cerebellar cortex. Cell Rep 17:3256-3268.

Turecek J, Jackman SL, Regehr WG (2017) Synaptotagmin 7 confers frequency invariance onto specialized depressing synapses. Nature 551:503506.

Uusisaari M, Knopfel T (2008) GABAergic synaptic communication in the GABAergic and non-GABAergic cells in the deep cerebellar nuclei. Neuroscience 156:537-549.

Uusisaari M, Obata K, Knopfel T (2007) Morphological and electrophysiological properties of GABAergic and non-GABAergic cells in the deep cerebellar nuclei. J Neurophysiol 97:901-911.

Vaden JH, Banumurthy G, Gusarevich ES, Overstreet-Wadiche L, Wadiche JI (2019) The readily-releasable pool dynamically regulates multivesicular release. Elife 8:e47434.

Valera AM, Doussau F, Poulain B, Barbour B, Isope P (2012) Adaptation of granule cell to Purkinje cell synapses to high frequency transmission. J Neurosci 32:3267-3280.

Walter JT, Khodakhah K (2009) The advantages of linear information processing for cerebellar computation. Proc Natl Acad Sci USA 106:44714476.

Welsh JP, Lang EJ, Suglhara I, Llinas R (1995) Dynamic organization of motor control within the olivocerebellar system. Nature 374:453-457.

Whitmire CJ, Stanley GB (2016) Rapid sensory adaptation redux: a circuit perspective. Neuron 92:298-315.

Xiao J, Cerminara NL, Kotsurovskyy Y, Aoki H, Burroughs A, Wise AK, Luo Y, Marshall SP, Sugihara I, Apps R, Lang EJ (2014) Systematic regional variations in Purkinje cell spiking patterns. PLoS One 9:e105633.

Xue R, Ruhl DA, Briguglio JS, Figueroa AG, Pearce RA, Chapman ER (2018) Doc2-mediated superpriming supports synaptic augmentation. Proc Natl Acad Sci USA 115:E5605-E5613.

Zhou H, Lin Z, Voges K, Ju C, Gao Z, Bosman LW, Ruigrok TJ, Hoebeek FE, De Zeeuw CI, Schonewille M (2014) Cerebellar modules operate at different frequencies. eLife 3:e02536.

Zucker RS, Regehr WG (2002) Short-term synaptic plasticity. Annu Rev Physiol 64:355-405. 